\title{
MOLECULAR ABUNDANCES AND LOW-MASS STAR FORMATION. I. Si- AND S-BEARING SPECIES TOWARD IRAS $16293-2422$
}

\author{
Geoffrey A. Blake, ${ }^{1}$ Ewine F. van Dishoeck, ${ }^{2}$ David J. Jansen, ${ }^{2}$ T. D. Groesbeck, ${ }^{3}$ and Lee G. Mundy ${ }^{4}$ \\ Received 1993 July 21; accepted 1993 December 22
}

\begin{abstract}
Results from millimeter and submillimeter spectral line surveys of the protobinary source IRAS $16293-2422$ are presented. Here we outline the abundances of silicon- and sulfur-containing species. A combination of rotation diagram and full statistical equilibrium/radiative transfer calculations is used to constrain the physical conditions toward IRAS 16293 and to construct its beam-averaged chemical composition over a $10-20^{\prime \prime}(1600-3200 \mathrm{AU})$ scale. The chemical complexity as judged by species such as $\mathrm{SiO}, \mathrm{OCS}$, and $\mathrm{H}_{2} \mathrm{~S}$, is intermediate between that of dark molecular clouds such as L134N and hot molecular cloud cores such as Orion KL. From the richness of the spectra compared to other young stellar objects of similar luminosity, it is clear that molecular abundances do not scale simply with mass; rather, the chemistry is a strong function of evolutionary state, i.e., age.
\end{abstract}

Subject headings: circumstellar matter - ISM: abundances - ISM: individual (IRAS 16293 - 2422) ISM: molecules

\section{INTRODUCTION}

The earliest stages of star formation occur deep within the interior of molecular clouds. Spectral line observations of active star-forming sites at radio, millimeter, and infrared wavelengths provide powerful probes of these regions and, when combined with theoretical models, have led to an improved understanding of the birth of stars (Lada 1991; Shu 1992). Many uncertainties remain, however. Chief among these is the role of chemistry. Clearly, the chemical composition of the interstellar medium responds to changes in physical parameters such as kinetic temperature, density, and radiation field, but it is not well known how much chemistry itself can influence the physical evolution of regions such as collapsing molecular cloud cores. For example, the abundances of low ionization potential species such as sulfur and other "metals" help maintain the fractional ionization of molecular cloud cores; yet they are poorly constrained observationally, and theoretical predictions are beset by uncertainties in processes such as gas-grain interactions (d'Hendecourt, Allamandola, \& Greenberg 1985; Brown \& Charnley 1990; Rawlings et al. 1992; Caselli, Hasegawa, \& Herbst 1993). The ionization fraction, in turn, controls the interactions of ions and neutrals with magnetic fields, thereby influencing directly the nature of the collapse (Hartquist et al. 1993).

At later stages, the chemical impact of young stars on the dense molecular clouds in which they are born can be substantial. High-mass stars, for example, inject massive amounts of energy into their surroundings, and the chemical modifications which result from this increased energy density have been noted observationally for quite some time. Indeed, sources such as Orion KL, Sagittarius B2, W51, and W3 have become the objects of intense study (Blake et al. 1987; Sutton et al.

\footnotetext{
${ }^{1}$ Division of Geological and Planetary Sciences, 170-25, California Institute of Technology, Pasadena, CA 91125.

${ }^{2}$ Leiden Observatory, P.O. Box 9513, 2300 RA Leiden, The Netherlands.

${ }^{3}$ Division of Physics, Mathematics and Astronomy, 320-47, California Institute of Technology, Pasadena, CA 91125.

4 Department of Astronomy, University of Maryland, College Park, MD 20742 .
}

1991; Helmich et al. 1994). For lower luminosity sources, less is known. Nevertheless, substantial amounts of warm, dense material are expected in these cores, and, as such, the chemical composition of low-mass young stellar objects (YSOs) is also expected to differ from regions of "pristine" molecular cloud material. The new generation of large-aperture, high-efficiency millimeter- and submillimeter-wave telescopes available at sites with high atmospheric transmission now enables chemical studies of star formation to be conducted over the full range of luminosity in the nearest molecular clouds.

Of the low-luminosity, and presumably low-mass, sources discovered to date, IRAS 16293-2422 stands as one of the best candidates for chemical study (Walker et al. 1986). It is apparently a young binary system, with two millimeter dust continuum sources, each of total mass $\sim 0.5 M_{\odot}$, at a projected separation of $840 \mathrm{AU}$ (position angle $135^{\circ}$; see Wootten 1989; Mundy et al. 1992). Located only 160 pc from the Sun, IRAS 16293 is an extreme class I YSO (Lada 1991). Indeed, no emission has been detected at wavelengths shorter than $20 \mu \mathrm{m}$, yet IRAS 16293 is a rich source of molecular line and dust continuum emission from centimeter to far-infrared wavelengthsespecially considering its modest luminosity of $27 L_{\odot}$. This, coupled with its proximity, has made IRAS 16293 a compelling source for both single-dish and interferometric observations (Mundy, Wootten, \& Wilking 1990; Walker et al. 1988, 1990). It has become for all intents and purposes the low-mass counterpart to Orion KL.

We have therefore undertaken systematic millimeter- and submillimeter-wave spectral line surveys of IRAS 16293-2422 in the $\lambda=1.3$ and $\lambda=0.87 \mathrm{~mm}$ atmospheric windows, whose goal is a comprehensive understanding of the chemical changes brought about by the physical forces involved in the formation of low-mass stars. Indeed, one of the tremendous advantages to spectral line studies at these wavelengths is their sensitivity to both warm, high-density and cold, low-density gas when a range of properly chosen molecules are observed or when unbiased surveys are acquired. Here we present the results for $\mathrm{Si}$ - and S-containing, species. In a subsequent paper we will outline our results for organic compounds such as $\mathrm{HCN} / \mathrm{HNC}$ and $\mathrm{CH}_{3} \mathrm{OH}$ (van Dishoeck et al. 1994). 
This partitioning reflects the fact that the differing properties of first- and second-row molecules can lead to chemical manifestations of a number of physical processes. In particular, the abundances of second-row species are often sensitive to the activation of chemical pathways that rely on energy densities well above those typically present in molecular cloud cores. Of the silicon- and sulfur-containing molecules, only $\mathrm{SiO}$ (and perhaps SiS) is truly refractory in a material sense, but for certain sulfur-containing species the gas-phase formation routes involve barriers which require kinetic temperatures in excess of $1000 \mathrm{~K}$ for reaction rates to become appreciable. Thus, while elemental $\mathrm{Si}$ and $\mathrm{S}$ have very different thermodynamic properties (Si tends to be strongly depleted even in lowextinction gas, while the $\mathrm{S}$ depletion is very modest [Jenkins 1987]), their gas-phase chemical behavior is similar - only a very small fraction of the cosmically available $\mathrm{Si}$ or $\mathrm{S}$ is typically observed in molecular species in dark clouds.

Shocks are often thought to provide the necessary driving force when larger amounts of Si- and S-bearing molecules are found (Neufeld \& Dalgarno 1989; van Dishoeck et al. 1992b; van Dishoeck, Jansen, \& Phillips 1993). This is not to say the formation routes of such species in shocks are the same. Indeed, the liberation of significant amounts of $\mathrm{Si}$ into the gas phase is likely to require extensive sputtering or outright destruction of dust grains; whereas formation of molecules such as $\mathrm{H}_{2} \mathrm{~S}$ is likely to proceed via gas phase chemistry in the post-shock cooling regions. The varying nature of interstellar shock waves (C- vs. J-type) can produce quite varied chemical responses, and this coupled with our poor understanding of gas-grain sulfur and silicon processes makes a detailed assessment of the roles of quiescent versus "energetic" chemistry difficult. These and other uncertainties are discussed at greater length below.

\section{OBSERVATIONS}

Spectral lines between 200 and $400 \mathrm{GHz}$ have been studied with the CSO ${ }^{5} 10.4 \mathrm{~m}$ and the JCMT $15 \mathrm{~m}$ telescopes located atop Mauna Kea, Hawaii. The facility 230 and $345 \mathrm{GHz}$ SIS double sideband heterodyne receivers were coupled to the facility $500 \mathrm{MHz}$ bandwidth AOS backends, which provide nominal resolutions of $1 \mathrm{MHz}$ (CSO) and $0.5 \mathrm{MHz}$ (JCMT). The actual resolution is often somewhat lower, and in selected cases where narrow self-absorption lines were found, the CSO $50 \mathrm{MHz}$ AOS was used to provide spectra at $100 \mathrm{kHz}$ resolution. Sideband assignments for the CSO data were confirmed by comparing spectra obtained with small local oscillator displacements, typically $10 \mathrm{MHz}$, but the relative sparseness of the emission is such that the identification of lines from known interstellar species is quite secure.

Most of the JCMT data were acquired during a single run in 1992 July with receivers A2 and B3i, with limited observations for comparison in 1991 February/March using receiver A1. The CSO data were obtained over several runs from 1989 May to 1991 July. In most cases the spectra were acquired in good weather and at moderate elevation angles, with doublesideband system temperatures ranging from approximately

\footnotetext{
${ }^{5}$ The Caltech Submillimeter Observatory is operated by the California Institute of Technology under funding from the U.S. National Science Foundation (AST 90-15755).

${ }^{6}$ The James Clerk Maxwell Telescope is operated by the Royal Observatory, Edinburgh, on behalf of the UK Science and Engineering Research Council, the Netherlands Organization for Pure Research, and the National Research Council of Canada.
}

500 to $2000 \mathrm{~K}$ in each atmospheric window. Emission-line strengths were calibrated at the telescope via the chopper wheel method (Kutner \& Ulich 1981) to yield $T_{A}^{*}$ values, which have then been converted into main beam brightness temperatures, $T_{\mathrm{MB}}=T_{A}^{*} / \eta_{\mathrm{MB}}$, using telescope efficiencies measured on Jupiter and Mars. The CSO main beam efficiencies $\left(\eta_{\mathrm{MB}}\right)$ are 0.72 and 0.60 at 230 and $345 \mathrm{GHz}$, while the JCMT efficiencies are $\eta_{\mathrm{MB}}(230 \mathrm{GHz})=0.7$ for the 1991 run and $\eta_{\mathrm{MB}}(230$ $\mathrm{GHz})=0.55$ and $\eta_{\mathrm{MB}}(345 \mathrm{GHz})=0.48$ for 1992 . Absolute calibration is accurate to $\sim 30 \%$, but the relative calibration within each data set is better $(\leq 15 \%)$. Typical integration times were 15-30 minutes on source, resulting in rms antenna temperatures of $20-40 \mathrm{mK}$.

Because the strongest lines toward IRAS 16293-2422 are about an order of magnitude weaker than toward Orion KL, an unbiased survey would be meaningful only when conducted down to about $20 \mathrm{mK} \mathrm{rms}$ or lower, which would require prohibitive amounts of telescope time. Instead, we have observed selected parts of the 230 and $345 \mathrm{GHz}$ windows which contain a range of molecular transitions, covering about $20 \mathrm{GHz}$ in each window. The $239-250$ and $338-347 \mathrm{GHz}$ regions were covered completely.

Beam sizes are essentially diffraction limited for the two telescopes and are 30" (CSO) and 21" (JCMT) at $230 \mathrm{GHz}$, decreasing to $20^{\prime \prime}$ and $15^{\prime \prime}$, respectively, at $345 \mathrm{GHz}$. Note that the CSO beam size at $345 \mathrm{GHz}$ is comparable to that of the JCMT at $230 \mathrm{GHz}$, a feature that will be important in the analysis. Azimuth switching by several arcminutes from the nominal source position of R.A.(1950) $=16^{\mathrm{h}} 29^{\mathrm{m}} 20^{\mathrm{s}} .90$, decl.(1950) $=-24^{\circ} 22^{\prime} 13^{\prime \prime} .0$ was most often employed to collect the spectra at the CSO, whereas most settings on the JCMT were collected by beam chopping with a $180^{\prime \prime}$ azimuth throw. No major differences in the position- and beam-switched spectra were observed, especially for the higher excitation lines of rare molecules. For very extended molecules such as the main isotopes of $\mathrm{CO}, \mathrm{CS}, \mathrm{HCN}$, and $\mathrm{HCO}^{+}$, position switching to a position known to be free of ${ }^{12} \mathrm{CO}$ emission was employed (Walker et al. 1988). For a number of molecules limited maps or five-point cross observations were made to constrain the spatial extent of the emission. Selected spectra illustrating the variety of kinematic profiles and spectral density of emission features towards IRAS 16293 are presented in Figures 1-5.

\section{RESULTS}

\subsection{Rotation Diagrams}

In the following sections we summarize the data obtained for several Si- and S-containing molecules and, where possible, include rotation diagrams which give-under assumptions of optically thin, thermalized emission (LTE) - estimates of the excitation and abundance of the various species via the equation

$$
\frac{3 k \int T_{\mathrm{MB}} d V}{8 \pi^{3} \mu^{2} v S}=\frac{N_{u}}{g_{u}}=\frac{N_{T}}{Q\left(T_{\mathrm{ROT}}\right)} e^{-E_{u} / k T_{\mathrm{ROT}}} .
$$

Here $\mu, v$, and $S$ are the transition dipole moment, frequency, and line strength; $N_{T}$ is the total column density; $T_{\mathrm{ROT}}$ is the LTE rotational temperature; $\boldsymbol{E}_{\boldsymbol{u}}$ is the upper state energy; and $Q\left(T_{\text {ROT }}\right)$ is the rotational partition function. The level degeneracies $g_{u}$, line strengths, and partition functions must be defined consistently, and for these analyses we have used a substantially augmented version of the JPL microwave, millimeter, 


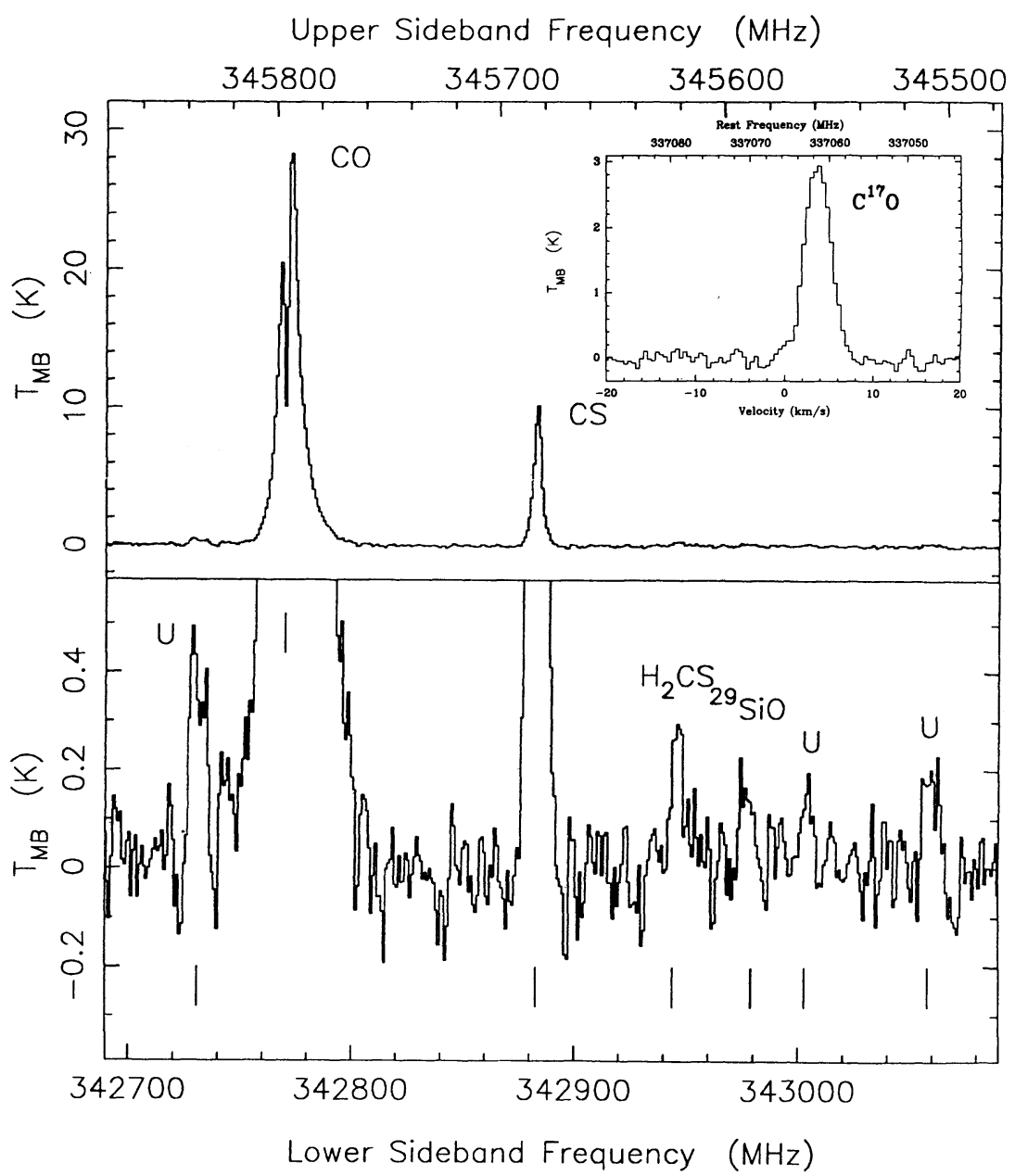

Fig. 1.- (Top panel) CSO double-sideband spectra of the $\mathrm{CO} J=3 \rightarrow 2$ (upper sideband, top legend) and CS $J=7 \rightarrow 6$ (lower sideband, lower legend) emission lines toward IRAS $16293-2422$. The insert presents a JCMT spectra of the $\mathrm{C}^{17} \mathrm{O} J=3 \rightarrow 2$ transition, which illustrates the high optical depth of the ${ }^{12} \mathrm{CO}$ feature. The deep self-absorption in the ${ }^{12} \mathrm{CO}$ transition is the result of the small offset used in the position switching to optimize the sensitivity to compact emission features. (Lower panel) An expanded sensitivity scale of the CSO CO/CS spectrum, revealing emission lines of other Si- and S-bearing species.

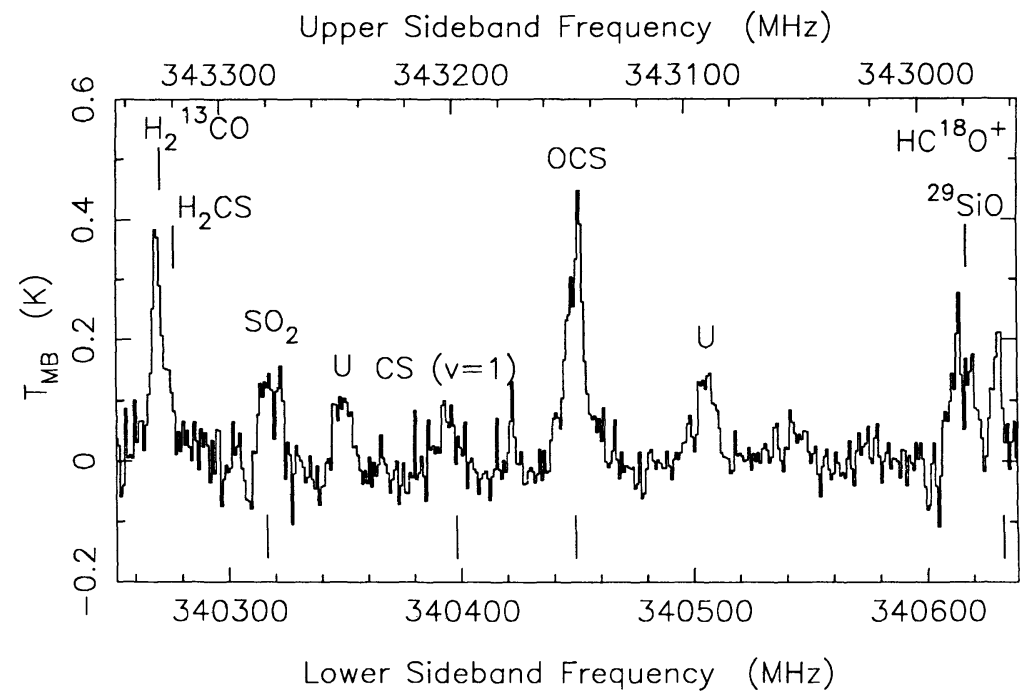

Fig. 2.-A CSO spectrum around the $\mathrm{CS} J=7 \rightarrow 6$ transition of vibrationally excited CS, which also contains emission from $\mathrm{OCS}, \mathrm{SO}{ }_{2}, \mathrm{H}_{2} \mathrm{CS}$, ${ }^{29} \mathrm{SiO}$, and $\mathrm{H}_{2}{ }^{13} \mathrm{CO}$. 


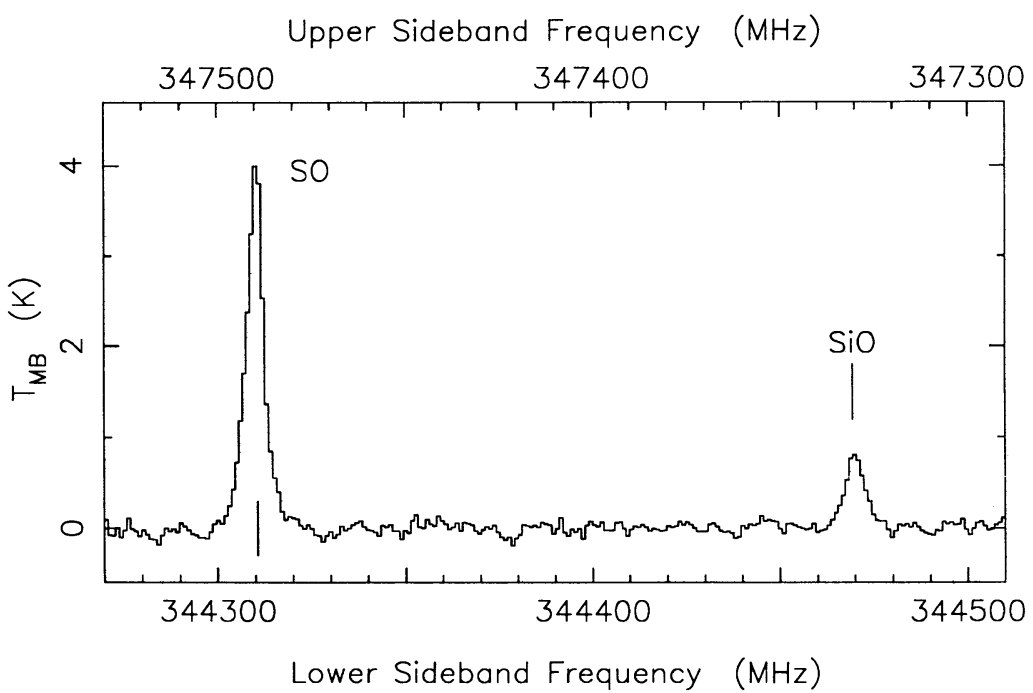

Fig. 3.-A CSO spectrum of the SO $N_{J}=8_{8} \rightarrow 77$ (lower sideband) and SiO $J=8 \rightarrow 7$ (upper sideband) transitions. Supersonic line wings are clearly visible on the $\mathrm{SO}$ emission (at this frequency, the small tick marks correspond to a velocity increment of $8.7 \mathrm{~km} \mathrm{~s}^{-1}$ ).

and submillimeter spectral line catalog available at the CSO (Poynter \& Pickett 1992; Groesbeck 1994) to construct the diagrams outlined below. Provided the lines are optically thin, the rotation diagram technique provides a lower bound to the true kinetic temperature, but it often overestimates the true column densities, since all levels are assumed to be thermalized, regardless of their excitation. We constructed rotation diagrams using either the observed integrated intensities or the peak temperatures with a fixed line width. The results of the two methods differ slightly on the weakest species but are consistent within the errors.

The combination of JCMT $230 \mathrm{GHz}$ and CSO $345 \mathrm{GHz}$ data is especially powerful due to the closely matched beam sizes and error patterns of the two telescopes at these frequencies, since the extent of the molecules is not known a priori but is found to be on the order of the beam size and to differ from molecule to molecule. We present next the LTE analyses of the Si- and S-bearing molecules detected in the CSO/JCMT surveys in sections which compare molecules with similar excitation and chemical characteristics.

\subsection{1. $\mathrm{CO}, \mathrm{CS}$}

Figure 1 presents a spectrum of the $\mathrm{CO} J=3 \rightarrow 2, \mathrm{C}^{17} \mathrm{O}$ $J=3 \rightarrow 2$, and CS $J=7 \rightarrow 6$ emission lines from IRAS $16293-2422$, which are illustrative of the large amount of warm, dense gas surrounding this source. The supersonic line wings of the main isotopic species arise from the extensive multipolar outflow, or outflows, from the protobinary source (Walker et al. 1988; Mizuno et al. 1990). A narrow selfabsorption feature is evident at the systemic velocity in the CO emission but is absent in the CS spectrum and arises from the cold, low-density $\rho$ Oph molecular cloud in which IRAS $16293-2422$ is embedded.

Table 1 summarizes the results obtained for the parent and

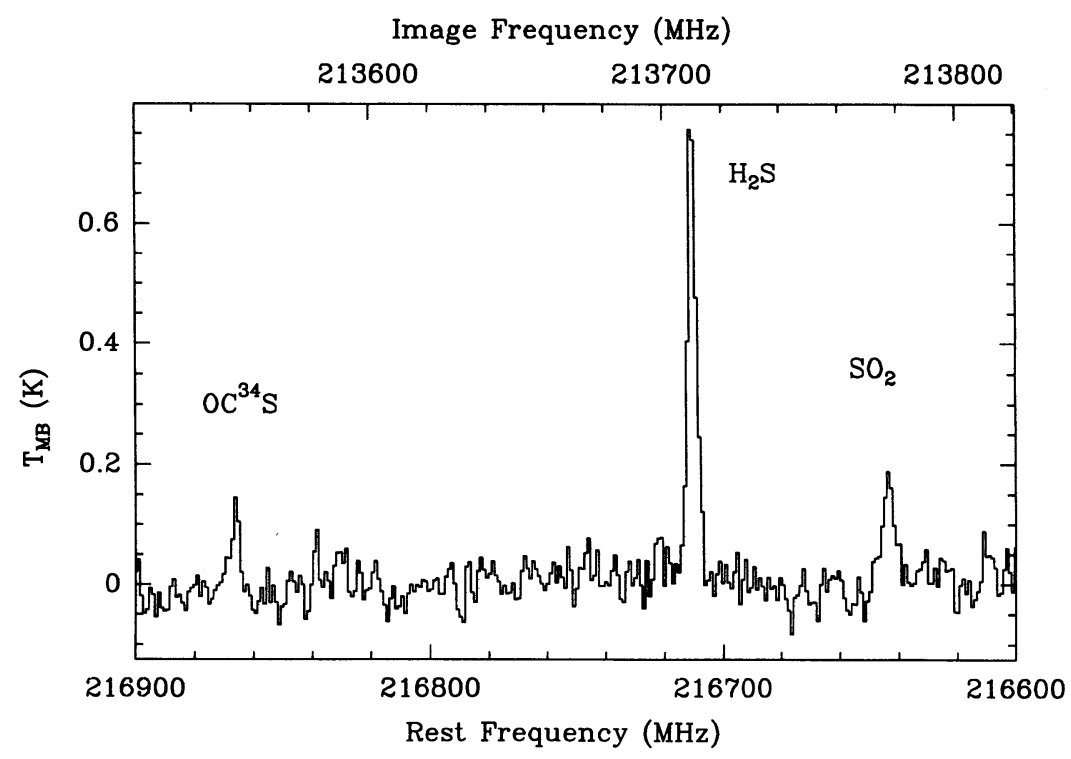

FIG. 4.-A JCMT spectrum near the $\mathrm{H}_{2} \mathrm{~S} 2_{20} \rightarrow 2_{11}$ transition, which also contains lines of $\mathrm{SO}_{2}$ and $\mathrm{OC}^{34} \mathrm{~S}$. The emission in Figs. 2 and 4 clearly indicates the optically thick character of the parent $\mathrm{OC}^{32} \mathrm{~S}$ lines. 


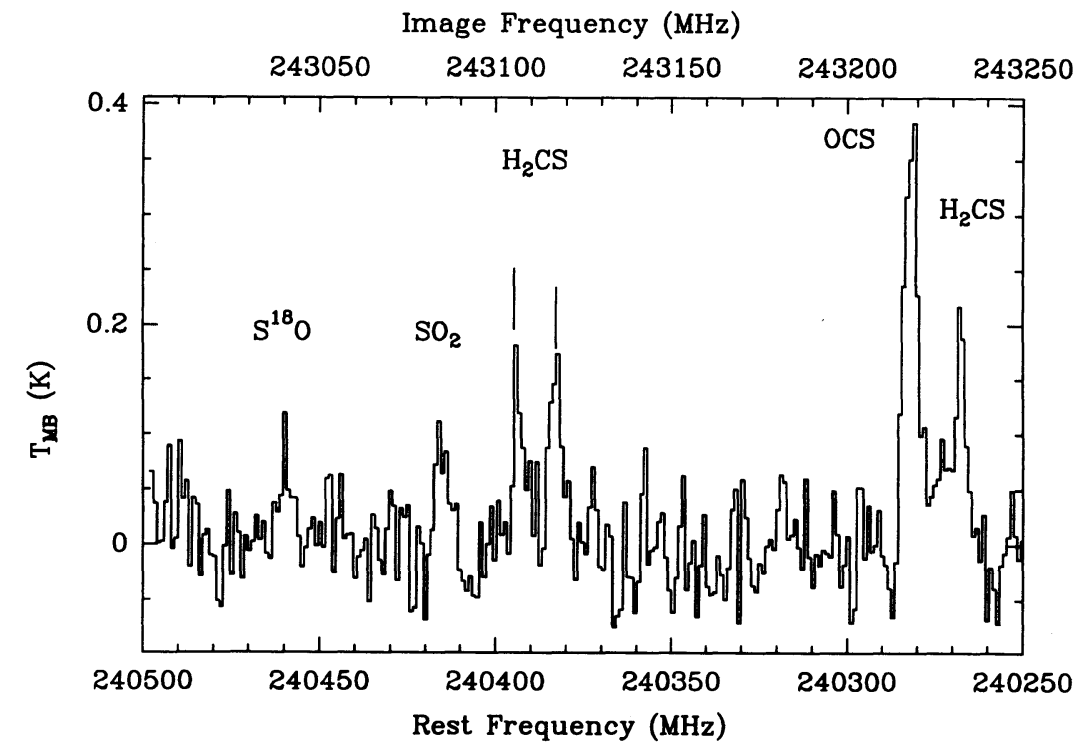

FIG. 5.-A JCMT spectrum near $240.5 \mathrm{GHz}$ (lower sideband), which contains the $\mathrm{H}_{2} \mathrm{CS} J_{K}=7_{K} \rightarrow 6_{K}$ manifold. Also visible are lines of $\mathrm{OCS}_{\text {and }} \mathrm{SO}$.

several isotropically substituted forms of $\mathrm{CO}$ and $\mathrm{CS}$. CO is included here as an "internal standard" for the abundance determinations that follow. The $\mathrm{C}^{\mathbf{1 8}} \mathrm{O}$ transitions are still marginally optically thick, so we use the $\mathrm{C}^{17} \mathrm{O}$ lines to derive beam-averaged LTE column densities. We find that $N\left(\mathrm{C}^{17} \mathrm{O}\right)=(5.0-7.5) \times 10^{15} \mathrm{~cm}^{-2}$ for $T_{\text {ROT }}=40-80 \mathrm{~K}$, which for $X\left(\mathrm{C}^{17} \mathrm{O}\right)=N\left(\mathrm{C}^{17} \mathrm{O}\right) / N\left(\mathrm{H}_{2}\right)=3.8 \times 10^{-8}$ (this abundance is obtained from the terrestrial isotopic ratio, that is, ${ }^{12} \mathrm{CO} /$ $\mathrm{C}^{17} \mathrm{O}=2674$, and a "canonical " $\mathrm{CO}$ abundance of $\mathrm{CO} / \mathrm{H}_{2}=$ $10^{-4}$; see Black \& Willner 1984; van Dishoeck et al. 1992a) yields a total column density of $N\left(\mathrm{H}_{2}\right)=(1.3-2.5) \times 10^{23}$ $\mathrm{cm}^{-2}$. The total mass in a $20^{\prime \prime}$ beam is $0.5-0.75 M_{\odot}$. The formal excitation temperature of the two $\mathrm{C}^{17} \mathrm{O}$ lines is $43 \mathrm{~K}$, close to the mean dust temperature of $40 \mathrm{~K}$ (Mundy, Wilking \& Myers 1986). Finally, the lack of wings in the isotopic CO transitions is illustrative of the considerably smaller column density of outflowing gas relative to the quiescent material.

The ${ }^{12} \mathrm{CO}$ main beam brightness temperature is $25 \mathrm{~K}$ in a $20^{\prime \prime}$ beam, which gives a lower limit of 0.625 for the area filling factor with $T_{\text {ROT }}=40 \mathrm{~K}$, or a source size of $\geq 15^{\prime \prime}$ (the lower limit arises from the self-absorbed nature of the $\mathrm{CO}$ profile).
The optically thick ${ }^{13} \mathrm{CO}$ source size is $10^{\prime \prime}$, as is that for the CS $5 \rightarrow 4$ and $7 \rightarrow 6$ emission for the same excitation temperature along with the measured telescope efficiencies and beam sizes. A similar source size is obtained from a $\mathrm{C}^{17} \mathrm{O} 3 \rightarrow 2 \mathrm{JCMT}$ half-beam sampled cross. An LTE analysis of the $C^{34} S$ peak temperatures and integrated line emission yields $T_{\text {ROT }}=37$ and $43 \mathrm{~K}$. The optically thin, thermalized column density is $N\left(C^{34} \mathrm{~S}\right)=1.1 \times 10^{13} \mathrm{~cm}^{-2}$, which for a terrestrial ${ }^{32} \mathrm{~S} /{ }^{34} \mathrm{~S}$ ratio of 22.5 gives a CS column density of $2.5 \times 10^{14} \mathrm{~cm}^{-2}$. If $C^{34} S J=3 \rightarrow 2$ data from the $30 \mathrm{~m}$ IRAM telescope in a similar beam are included (Menten et al. 1987), the LTE rotational temperature drops to $30 \mathrm{~K}$, and the $\mathrm{C}^{34} \mathrm{~S}$ column density increases to $1.5 \times 10^{13} \mathrm{~cm}^{-2}$.

Vibrationally excited CS may also have been detected through its $J=7 \rightarrow 6$ transition at $340.398 \mathrm{GHz}$, as seen in Figure 2, which also contains emission from a variety of other molecules. The high line density observed demands that several additional $J$ values be confirmed for the identification to be considered secure. If the detected feature is due to vibrationally excited CS, the excitation mechanism for this state is likely to be radiative pumping in gas close to the YSOs (Black 1993).

TABLE 1

OBSERVATIONS OF CO AND CS AND ISOTOPES

\begin{tabular}{|c|c|c|c|c|c|c|}
\hline Molecule & Line & $\begin{array}{l}\text { Frequency } \\
\text { (MHz) }\end{array}$ & $\int_{\left(\mathrm{K} \mathrm{km} \mathrm{s}^{-1}\right)} T_{\mathrm{MB}} d V$ & $\begin{array}{l}T_{\mathrm{MB}} \\
(\mathrm{K})\end{array}$ & $\begin{array}{c}\Delta V \\
\left(\mathrm{~km} \mathrm{~s}^{-1}\right)\end{array}$ & Telescope \\
\hline \multirow[t]{2}{*}{$\mathrm{CO} \ldots \ldots \ldots \ldots \ldots$} & $2-1$ & 230538.0 & 189.1 & $\ldots$ & 13.9 & $\mathrm{JCMT}^{\mathrm{a}}$ \\
\hline & $3-2$ & 345796.0 & 261.0 & $\ldots$ & 12.6 & $\mathrm{CSO}^{\mathrm{a}}$ \\
\hline${ }^{13} \mathrm{CO}$ & $3-2$ & 330588.1 & 43.3 & 9.05 & 4.50 & CSO \\
\hline $\mathrm{C}^{18} \mathrm{O}$ & $2-1$ & 219560.3 & 16.4 & 6.12 & 2.52 & JCMT \\
\hline \multirow{2}{*}{$\mathrm{C}^{17} \mathrm{O} \ldots \ldots \ldots \ldots$} & $2-1$ & 224714.4 & 7.1 & 2.50 & 2.67 & JCMT \\
\hline & $3-2$ & 337061.1 & 11.0 & 3.02 & 3.41 & JCMT \\
\hline${ }^{13} \mathrm{C}^{18} \mathrm{O}$ & $3-2$ & 314119.6 & $\ldots$ & $\leq 0.35$ & $\ldots$ & JCMT \\
\hline${ }^{13} \mathrm{C}^{17} \mathrm{O} \ldots \ldots \ldots \ldots$ & $2-1$ & 214574.1 & & $\leq 0.09$ & $\ldots$ & JCMT \\
\hline \multirow[t]{2}{*}{ CS $\ldots \ldots \ldots \ldots \ldots$} & $5-4$ & 244935.6 & 27.1 & 7.44 & 3.43 & $\mathrm{JCMT}^{\mathrm{a}}$ \\
\hline & $7-6$ & 342883.0 & 26.2 & 6.55 & 3.77 & CSO \\
\hline \multirow[t]{2}{*}{$\mathrm{C}^{34} \mathrm{~S} \ldots \ldots \ldots \ldots$} & $5-4$ & 241016.2 & 3.56 & 0.95 & 3.52 & JCMT \\
\hline & $7-6$ & 337396.7 & 3.68 & 0.87 & 3.97 & CSO \\
\hline $\operatorname{CS}(v=1) \ldots \ldots \ldots$ & $7-6$ & 340398.1 & 0.73 & 0.18 & 3.91 & CSO \\
\hline
\end{tabular}

${ }^{\text {a }}$ Self-absorption and wings present. 
TABLE 2

OBSERVATIONS OF $\mathrm{SO}, \mathrm{SiO}, \mathrm{SiS}$ AND ISOTOPES

\begin{tabular}{|c|c|c|c|c|c|c|}
\hline Molecule & Line & $\begin{array}{c}\text { Frequency } \\
\text { (MHz) }\end{array}$ & $\underset{\left(\mathrm{K} \mathrm{km} \mathrm{s}^{-1}\right)}{T_{\mathrm{MB}} d V}$ & $\begin{array}{l}T_{\mathrm{MB}} \\
(\mathbf{K})\end{array}$ & $\begin{array}{c}\Delta V \\
\left(\mathrm{~km} \mathrm{~s}^{-1}\right)\end{array}$ & Telescope \\
\hline \multirow[t]{11}{*}{ SO $\ldots \ldots \ldots \ldots \ldots$} & $87_{7}-7_{7}$ & 214357.0 & 0.76 & 0.24 & 2.91 & JCMT \\
\hline & $55-4$ & 215220.6 & 15.1 & 3.42 & 4.12 & $\mathrm{JCMT}^{\mathrm{a}}$ \\
\hline & $6_{5}-5_{4}$ & 219949.4 & 21.0 & 4.87 & 4.05 & $\mathrm{JCMT}^{\mathrm{a}}$ \\
\hline & $32-2$ & 246404.7 & $\ldots$ & $\leq 0.13$ & $\ldots$ & JCMT \\
\hline & $6_{6}-5_{5}$ & 258255.8 & 16.2 & 3.33 & 4.58 & $\mathrm{JCMT}^{\mathbf{a}}$ \\
\hline & $67-5$ & 261843.7 & 22.2 & 5.24 & 3.98 & $\mathrm{JCMT}^{\mathbf{a}}$ \\
\hline & $87_{7}-7_{6}$ & 340714.2 & 20.7 & 3.82 & 5.12 & CSO \\
\hline & $88-7$ & 344310.6 & 18.1 & 3.40 & 5.01 & JCMT \\
\hline & $88-7$ & 344310.6 & 22.8 & 3.88 & 5.54 & $\mathrm{CSO}$ \\
\hline & $89-78$ & 346528.5 & 37.2 & 6.71 & 5.2 & $\mathrm{CSO}^{\mathrm{a}, \mathrm{b}}$ \\
\hline & $7_{6}-67$ & 361351.2 & $\ldots$ & $\leq 0.5$ & $\ldots$ & $\mathrm{CSO}$ \\
\hline \multirow[t]{5}{*}{${ }^{34}$ SO ............. } & $3_{2}-23$ & 246152.3: & $\ldots$ & $\leq 0.13$ & $\ldots$ & JCMT \\
\hline & $65-5_{4}^{3}$ & 246663.4 & 1.20 & 0.22 & 5.00 & JCMT \\
\hline & $88_{7}-7_{6}$ & 337582.2 & 1.60 & 0.35 & 4.27 & $\mathrm{CSO}$ \\
\hline & $8_{9}-7_{8}$ & 339857.6 & 3.23 & 0.63 & 4.88 & $\mathrm{CSO}$ \\
\hline & $8_{9}-7_{8}^{\circ}$ & 339857.6 & 2.98 & 0.60 & 4.67 & $\mathrm{JCMT}^{\mathrm{c}}$ \\
\hline \multirow[t]{3}{*}{$S^{18} \mathrm{O} \ldots \ldots \ldots \ldots$} & $6_{6}-5_{5}$ & 239128.5 & $\ldots$ & $\leq 0.13$ & $\ldots$ & JCMT \\
\hline & $6_{7}-5_{6}$ & 243039.3 & $\ldots$ & 0.11 : & $\ldots$ & $\mathbf{J C M T}^{\mathbf{d}}$ \\
\hline & $3_{2}-2_{3}$ & 245630.5: & $\ldots$ & $\leq 0.11$ & $\ldots$ & JCMT \\
\hline \multirow{3}{*}{$\mathrm{SO}(v=1) \ldots \ldots \ldots$} & $88_{7}-7_{7}$ & 211327.6: & $\ldots$ & $\leq 0.11$ & $\ldots$ & JCMT \\
\hline & $5_{5}-4$ & 213501.0 & $\ldots$ & $\leq 0.09$ & $\ldots$ & JCMT \\
\hline & $65-5_{4}^{4}$ & 249646.7 & $\ldots$ & $\leq 0.09$ & $\ldots$ & JCMT \\
\hline \multirow[t]{4}{*}{$\mathrm{SiO} \ldots \ldots \ldots \ldots \ldots$} & $5-4$ & 217104.9 & 4.47 & 0.93 & 4.57 & JCMT \\
\hline & $6-5$ & 260518.0 & 3.77 & 0.96 & 3.7: & $\mathrm{JCMT}^{\mathrm{d}}$ \\
\hline & $8-7$ & 347330.6 & 4.52 & 0.78 & 5.38 & $\mathrm{CSO}$ \\
\hline & $8-7$ & 347330.6 & 5.79 & 0.90 & 6.10 & JCMT \\
\hline \multirow[t]{3}{*}{${ }^{29} \mathrm{SiO} \ldots \ldots \ldots \ldots$} & $5-4$ & 214385.0 & $\ldots$ & $\leq 0.07$ & $\ldots$ & JCMT \\
\hline & $6-5$ & 257255.0 & 0.58 & 0.18 & 3.08 & JCMT \\
\hline & $8-7$ & 342979.1 & 0.95 & 0.18 & 4.80 & $\mathrm{CSO}$ \\
\hline \multirow{2}{*}{ SiS $\ldots \ldots \ldots \ldots \ldots$} & $13-12$ & 235961.1 & $\ldots$ & $\leq 0.18$ & $\ldots$ & JCMT \\
\hline & $17-16$ & 308515.6 & $\ldots$ & $\leq 0.13$ & $\ldots$ & JCMT \\
\hline \multirow[t]{2}{*}{${ }^{29} \mathrm{SiS} \ldots \ldots \ldots \ldots \ldots$} & $12-11$ & 213815.4 & $\ldots$ & $\leq 0.07$ & $\ldots$ & JCMT \\
\hline & $14-13$ & 249434.3 & $\ldots$ & $\leq 0.13$ & $\ldots$ & JCMT \\
\hline
\end{tabular}

a Wings present.

b Blend with $\mathrm{SO}_{2}$.

c Blend with $\mathrm{CH}_{3} \mathrm{OH}$.

d Colon indicates uncertain value.

\subsection{2. $\mathrm{SiO}, \mathrm{SiS}, \mathrm{SO}$}

Line emission from the diatomic molecules $\mathrm{SiO}$ and $\mathrm{SO}$ is summarized in Table 2, which also presents upper limits for $\mathrm{SiS} .{ }^{28} \mathrm{SiO}$ emission, shown in Figure 3, is optically thick as judged by the emission detected in several lines of ${ }^{29} \mathrm{SiO}$. The integrated ${ }^{29} \mathrm{SiO}$ line emission yields a total column density of $2.0 \times 10^{12} \mathrm{~cm}^{-2}$ and an excitation temperature of several hundred degrees, whereas the ${ }^{29} \mathrm{SiO}$ peak temperatures yield $T_{\text {ROT }} \geq 60 \mathrm{~K}$, with a column density of $N\left({ }^{29} \mathrm{SiO}\right)=1.1 \times 10^{12}$ $\mathrm{cm}^{-2}$ for an assumed line width of $5 \mathrm{~km} \mathrm{~s}^{-1}$ (FWHM). This produces a total SiO abundance of $N(\mathrm{SiO})=2.2 \times 10^{13} \mathrm{~cm}^{-2}$, provided the terrestrial isotopic ratio is appropriate $\left({ }^{28} \mathrm{Si} /\right.$ ${ }^{29} \mathrm{Si}=19.7 ;{ }^{30} \mathrm{SiO}$ was not searched for). The LTE excitation temperature and parent emission strength yields an estimated source size of $\leq 3^{\prime \prime}-4^{\prime \prime}$.

The SO emission clearly has both compact and extended components. Simultaneous mapping of the SO $5_{5} \rightarrow 4_{4}$ and $\mathrm{H}_{2} \mathrm{CO} 3_{03} \rightarrow 2_{02}$ transitions in the $30^{\prime \prime} \mathrm{CSO}$ beam over a $3^{\prime} \times 2^{\prime}$ area reveals a compact, but clearly extended source at the position of IRAS 16293 as well as emission at a position $60^{\prime \prime}$ due east in the blue lobe of the main outflow (Mizuno et al. 1990). No SO emission is observed elsewhere. The extension at IRAS 16293 itself is nearly E-W. At $3^{\prime \prime}$ resolution, millimeter interferometric images reveal unresolved SO emission centered on the southeastern dust continuum source, plus weaker red and blue emission $\mathrm{E}$ and $\mathrm{W}$ that is anticorrelated with the velocity sense of the E-W outflow (Mundy et al. 1992) but which does follow the rotational velocity pattern of the source inferred from interferometric images of $\mathrm{C}^{18} \mathrm{O}$ and $\mathrm{NH}_{3}$ (Mundy et al. 1990). ${ }^{32} \mathrm{SO}$ is marginally optically thick toward IRAS 16293, and supersonic line wings are visible on the strongest lines (see Fig. 3). The ${ }^{34} \mathrm{SO}$ rotation diagram is best fitted with $T_{\text {ROT }}\left({ }^{34} \mathrm{SO}\right) \sim 80 \mathrm{~K}, N\left({ }^{34} \mathrm{SO}\right)=3.5 \times 10^{13} \mathrm{~cm}^{-2}$ implying a parent (that is, ${ }^{32} \mathrm{SO}$ ) column density of $7.9 \times 10^{14}$ $\mathrm{cm}^{-2}$. This is about a factor of 2 larger than that found from a rotation diagram of SO itself. The combination of the peak temperatures from Table 2 with the rotation diagram analysis yields a formal source size of 7"-8".

\subsection{3. $\mathrm{OCS}$ and $\mathrm{HCS}^{+}$}

Like $\mathrm{SiO}$, OCS is optically thick and rather warm, as is evidenced by the intensities of the isotopically substituted species presented in Figure 4 and Table 3. The column density of $\mathrm{OC}^{34} \mathrm{~S}$ is $6.3 \times 10^{13} \mathrm{~cm}^{-2}$, and its excitation temperature is approximately $80 \mathrm{~K}$. A column density of $1.4 \times 10^{15} \mathrm{~cm}^{-2}$ is found for the parent isotopomer, nearly a factor of 7 greater than that predicted by an LTE analysis of the OCS emission. $\mathrm{O}^{13} \mathrm{CS}$ produces a greater abundance still for a ${ }^{12} \mathrm{C} /{ }^{13} \mathrm{C}$ ratio of 60 (Langer \& Penzias 1990). From these measurements a source size of $\leq 2^{\prime \prime}-3^{\prime \prime}$ is deduced. 
TABLE 3

OBSERVATIONS OF $\mathrm{HCS}^{+}$AND OCS AND ISOTOPES

\begin{tabular}{ccccccc}
\hline Molecule & Line & $\begin{array}{c}\text { Frequency } \\
(\mathrm{MHz})\end{array}$ & $\begin{array}{c}\int T_{\mathrm{MB}} d V \\
\left(\mathrm{~K} \mathrm{~km} \mathrm{~s}^{-1}\right)\end{array}$ & $\begin{array}{c}T_{\mathrm{MB}} \\
(\mathrm{K})\end{array}$ & $\begin{array}{c}\Delta V \\
\left(\mathrm{~km} \mathrm{~s}^{-1}\right)\end{array}$ & Telescope \\
\hline HCS $^{+} \ldots \ldots \ldots \ldots$ & $5-4$ & 213360 & 0.82 & 0.18 & 4.15 & JCMT \\
& $8-7$ & 341350.8 & 1.75 & 0.23 & 7.19 & CSO \\
$\mathrm{OCS} \ldots \ldots \ldots \ldots$. & $20-19$ & 243218.0 & 2.22 & 0.38 & 5.48 & JCMT \\
& $28-27$ & 340449.2 & 2.52 & 0.38 & 6.22 & CSO \\
$\mathrm{OC}^{34} \mathrm{~S} \ldots \ldots \ldots \ldots$. & $18-17$ & 213553 & 0.64 & 0.15 & 4.00 & JCMT \\
& $21-20$ & 249133 & 0.87 & 0.16 & 5.16 & JCMT \\
& $22-21$ & 260992 & 0.71 & 0.12 & 5.59 & JCMT \\
$\mathrm{O}^{13} \mathrm{CS} \ldots \ldots \ldots \ldots$ & $18-17$ & 218199.0 & 0.33 & 0.12 & 2.49 & JCMT \\
& $20-19$ & 242435.4 & 0.87 & 0.14 & $6.0:$ & JCMT \\
& $21-20$ & 254552.7 & $\ldots$ & $\leq 0.11$ & $\ldots$ & JCMT \\
${ }^{18} \mathrm{OCS} \ldots \ldots \ldots \ldots$ & $19-18$ & 216753 & $\ldots$ & $\leq 0.07$ & $\ldots$ & JCMT \\
\hline
\end{tabular}

${ }^{a}$ Blend with ${ }^{18}$ OCS 21-20.

The peak main beam brightness temperatures of the two $\mathrm{HCS}^{+}$transitions detected formally yield $T_{\mathrm{ROT}}\left(\mathrm{HCS}^{+}\right) \approx 60 \mathrm{~K}$ with large formal errors. With an assumed line width of $\Delta V(\mathrm{FWHM})=4.5 \mathrm{~km} \mathrm{~s}^{-1}$, the LTE column density is $2.8 \times 10^{12} \mathrm{~cm}^{-2}$ with the peak temperature data. Raw integrated line emission yields both higher temperature and column density, $T_{\text {ROT }}=230 \mathrm{~K}$ and $N\left(\mathrm{HCS}^{+}\right)=5.0 \times 10^{12}$ $\mathrm{cm}^{-2}$. The spectra of isotopically substituted forms of $\mathrm{HCS}^{+}$ have not been recorded in the laboratory.

\subsection{4. $\mathrm{H}_{2} \mathrm{~S}$ and $\mathrm{H}_{2} \mathrm{CS}$}

A single line of $\mathrm{H}_{2} \mathrm{~S}$, the $2_{20} \rightarrow 2_{11}$ transition shown in Figure 4, has been detected. For excitation temperatures between 30 and $100 \mathrm{~K}$, the LTE column density varies by less than $50 \%$ and is found to be approximately $3.0 \times 10^{14} \mathrm{~cm}^{-2}$.

$\mathrm{H}_{2} \mathrm{CS}$ is a nearly prolate symmetric top, and several of the $K_{p}$ ladders are contained within a single double-sideband spectrum, as is shown in Figure 5. The net result is very good relative intensity information. The full $\mathrm{H}_{2} \mathrm{CS}$ data set is summarized in Table 4 , and the rotation diagram is shown in Figure 6. We find $T_{\mathrm{ROT}}\left(\mathrm{H}_{2} \mathrm{CS}\right) \approx 60 \pm 10 \mathrm{~K}$ and $\mathrm{N}\left(\mathrm{H}_{2} \mathrm{CS}\right) \approx$ $3.7 \times 10^{13} \mathrm{~cm}^{-2}$. Upper limits for $\mathrm{H}_{2}{ }^{34} \mathrm{CS}$ constrain the optical depth in the parent species to be $\leq 4$.

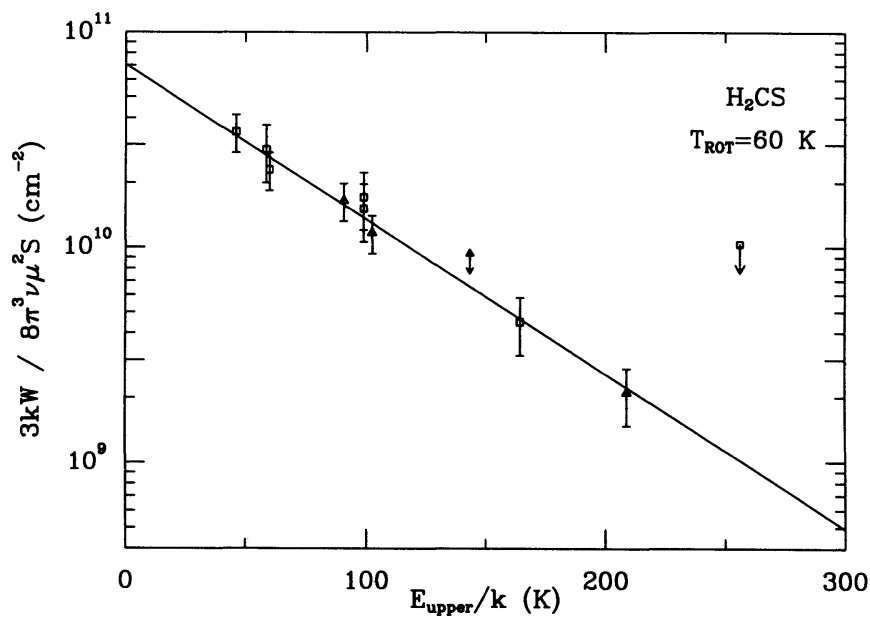

FIG. 6.-A rotation diagram plot of the CSO/JCMT data for $\mathrm{H}_{2} \mathrm{CS}$. The JCMT data points are presented as squares, while the CSO data are labeled with a triangle. The errors bars are $20 \%$ of the line intensities or $30 \%$ in the case of the weakest lines. Upper limits are denoted by arrows.

TABLE 4

OBSERVATIONS OF $\mathrm{H}_{2} \mathrm{~S}$ AND $\mathrm{H}_{2} \mathrm{CS}$

\begin{tabular}{|c|c|c|c|c|c|c|}
\hline Molecule & Line & $\begin{array}{c}\text { Frequency } \\
(\mathrm{MHz})\end{array}$ & $\int_{\left(\mathrm{K} \mathrm{km} \mathrm{s}^{-1}\right)}^{T_{\mathrm{MB}} d V}$ & $\begin{array}{l}T_{\mathrm{MB}} \\
(\mathrm{K})\end{array}$ & $\begin{array}{c}\Delta V \\
\left(\mathrm{~km} \mathrm{~s}^{-1}\right)\end{array}$ & Telescope \\
\hline $\begin{array}{l}\mathbf{H}_{2} \mathrm{C}^{34} \mathrm{~S} \ldots \ldots \ldots \ldots \\
\mathrm{H}_{2}{ }^{13} \mathrm{CS} \ldots \ldots \ldots \ldots\end{array}$ & $\begin{array}{c}2_{2,0}-2_{1,1} \\
7_{1,7}-6_{1,6} \\
7_{6,2,1}-6_{6,1 / 0} \\
7_{0,7}-6_{0,6} \\
7_{4,4 / 3}-6_{4,3 / 2} \\
7_{2,6}-6_{2,5} \\
7_{3,5 / 4}-6_{3,4 / 3} \\
7_{2,5}-6_{2,4} \\
7_{1,6}-6_{1,5} \\
10_{1,10}-9_{1,9} \\
10_{1,10}-9_{1,9} \\
10_{0,10}-9_{0,9} \\
10_{2,9}-9_{2,8} \\
10_{3,8}-9_{3,7} \\
7_{0,7}-6_{0,6} \\
7_{1,6}-6_{1,5} \\
8_{1,8}-7_{1,7}\end{array}$ & $\begin{array}{l}216710.4 \\
236726.3 \\
240178.0 \\
240266.2 \\
240331.4 \\
240381.3 \\
240392.3 \\
240548.3 \\
244047.8 \\
338080.8 \\
338080.8 \\
342944.0 \\
343319.6 \\
343408.1 \\
236198.8 \\
239858.5 \\
260310.3\end{array}$ & $\begin{array}{l}3.91 \\
2.00 \\
\ldots \\
0.86 \\
\ldots \\
0.40 \\
0.55 \\
0.31 \\
2.15 \\
2.40 \\
3.03 \\
0.92 \\
\leq 0.50 \\
0.77 \\
\ldots \\
\ldots \\
\ldots\end{array}$ & $\begin{array}{r}0.80 \\
0.53 \\
\leq 0.11 \\
0.22 \\
0.09: \\
0.10 \\
0.14 \\
0.09 \\
0.44 \\
0.56 \\
0.45 \\
0.22 \\
0.25 \\
0.15 \\
\leq 0.18 \\
\leq 0.11 \\
\leq 0.18\end{array}$ & $\begin{array}{c}4.65 \\
3.53 \\
\ldots \\
3.80 \\
\ldots \\
3.61 \\
3.64 \\
3.27 \\
4.67 \\
3.99 \\
6.37 \\
4.0 \\
1.88 \\
4.94 \\
\ldots \\
\ldots \\
\ldots\end{array}$ & 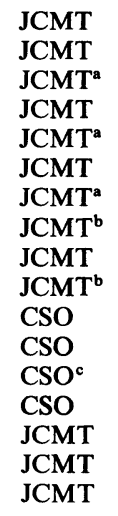 \\
\hline
\end{tabular}

alend of two lines.

b Bad baseline.

${ }^{c}$ Blend with $\mathrm{H}_{2}{ }^{13} \mathrm{CO}$ 
TABLE 5

OBSERVATIONS OF $\mathrm{SO}_{2}$

\begin{tabular}{|c|c|c|c|c|c|c|}
\hline Molecule & Line & $\begin{array}{l}\text { Frequency } \\
(\mathrm{MHz})\end{array}$ & $\underset{\left(\mathbf{K ~ k m ~ s} ~^{-1}\right)}{T_{\mathrm{MB}} d V}$ & $\begin{array}{l}T_{\mathrm{MB}} \\
(\mathbf{K})\end{array}$ & $\begin{array}{c}\Delta V \\
\left(\mathrm{~km} \mathrm{~s}^{-1}\right)\end{array}$ & Telescope \\
\hline & $\begin{array}{c}.26_{3,23}-26_{2,24} \\
22_{2,20}-22_{1,21} \\
22_{7,15}-23_{6,18} \\
11_{1,11}-10_{0,10} \\
4_{2,2}-3_{1,3} \\
16_{1,15}-15_{2,14} \\
21_{7,15}-22_{6,16} \\
18_{1,17}-18_{0,18} \\
5_{2,4}-4_{1,3} \\
5_{4,2}-6_{3,3} \\
14_{0,14}-13_{1,13} \\
26_{3,23}-25_{4,22} \\
10_{3,7}-10_{2,8} \\
15_{2,14}-15_{1,15} \\
10_{5,5}-11_{4,8} \\
13_{1,13}-12_{0,12} \\
8_{3,5}-8_{2,6} \\
7_{3,5}-7_{2,6} \\
9_{3,7}-9_{2,8} \\
30_{4,26}-30_{3,27} \\
21_{2,20}-21_{1,21} \\
4_{3,1}-3_{2,2} \\
8_{2,6}-7_{1,7} \\
18_{4,14}-18_{3,15} \\
20_{4,19}-19_{2,18} \\
28_{2,26}-28_{1,27} \\
13_{2,12}-12_{1,11} \\
16_{4,12}-16_{3,13} \\
19_{1,19}-18_{0,18} \\
5_{3,3}-6_{0,6} \\
14_{4,10}-13_{3,11} \\
13_{4,10}-13_{3,11} \\
15_{4,12}-15_{3,13} \\
15_{4,12}-15_{3,13} \\
11_{4,8}-11_{3,9} \\
11_{4,8}-11_{3,9}\end{array}$ & $\begin{array}{l}213068.4 \\
216643.3 \\
219276.0 \\
221965.2 \\
235151.7 \\
236216.7 \\
238992.6 \\
240942.8 \\
241615.8 \\
243087.7 \\
244254.2 \\
245339.4 \\
245563.4 \\
248057.4 \\
248830.8 \\
251199.7 \\
251210.6 \\
257100.0 \\
258942.2 \\
259599.5 \\
332091.4 \\
332505.2 \\
334673.3 \\
338306.0 \\
338611.8 \\
340316.4 \\
345338.5 \\
346523.9 \\
346652.2 \\
348633.3 \\
351873.8 \\
357165.4 \\
357241.2 \\
357241.2 \\
357387.6 \\
357387.6\end{array}$ & 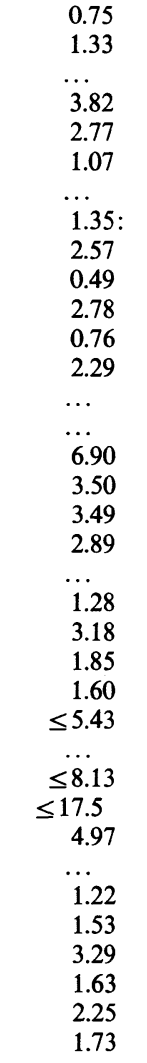 & $\begin{array}{r}0.15 \\
0.17 \\
0.04: \\
0.86 \\
0.67 \\
0.29 \\
\leq 0.13 \\
0.12 \\
0.60 \\
0.10 \\
0.62 \\
0.12 \\
0.46 \\
0.4: \\
\leq 0.13 \\
1.09 \\
0.63 \\
0.64 \\
0.58 \\
\leq 0.15 \\
0.30 \\
0.58 \\
0.33 \\
0.25 \\
0.73 \\
\leq 0.15 \\
1.22 \\
5.00 \\
0.77 \\
\leq 0.12 \\
0.23 \\
0.33 \\
0.40 \\
0.23 \\
0.35 \\
0.28\end{array}$ & $\begin{array}{c}4.78 \\
7.17 \\
\ldots .18 \\
4.18 \\
3.87 \\
3.56 \\
\ldots . \\
10.55: \\
4.06 \\
4.82 \\
4.25 \\
5.99 \\
4.81 \\
\ldots \\
\ldots \\
5.97 \\
5.24 \\
5.21 \\
4.64 \\
\ldots \\
3.95 \\
5.13 \\
5.19 \\
5.94 \\
6.99 \\
\ldots \\
6.28 \\
3.28 \\
6.14 \\
\ldots \\
4.90 \\
4.29 \\
7.95 \\
6.74 \\
5.85 \\
5.83\end{array}$ & $\begin{array}{l}\text { JCMT } \\
\text { JCMT } \\
\text { JCMT } \\
\text { JCMT } \\
\text { JCMT } \\
\text { JCMT } \\
\text { JCMT } \\
\text { JCMT } \\
\text { JCMT } \\
\text { JCMT } \\
\text { JCMT }^{\mathrm{b}} \\
\text { JCMT }^{\text {JCMT }} \\
\text { JCMT } \\
\text { JCMT } \\
\text { JCMT } \\
\text { JCMT } \\
\text { JCMT } \\
\text { JCMT } \\
\text { JCMT } \\
\text { CSO } \\
\text { CSO } \\
\text { CSO } \\
\text { CSO } \\
\text { CSO } \\
\text { CSO } \\
\text { CSO } \\
\text { CSO } \\
\text { CSO } \\
\text { CSO } \\
\text { CSO } \\
\text { CSO } \\
\text { JCMT } \\
\text { CSO } \\
\text { JCMT } \\
\text { CSO }\end{array}$ \\
\hline
\end{tabular}

NoTE.-A colon indicates an uncertain value.

a Blend with CCD.

b At edge spectrum.

c Blend with CS wing.

d Blend with $\mathrm{SO}_{2} 9_{37}-9_{28}$.

e Blend with $\mathrm{CH}_{3} \mathrm{OH}$.

f Blend with $\mathrm{H}^{13} \mathrm{CN}$.

g Blend with SO.

3.1.5. $\mathrm{SO}_{2}$

Along with $\mathrm{H}_{2} \mathrm{CS}, \mathrm{SO}_{2}$ is the only sulfur- and siliconcontaining species for which an extensive rotation diagram can be generated (see Table 5 and Fig. 7). The formal fits yield $T_{\text {ROT }}=95 \pm 5 \mathrm{~K}$ and $N\left(\mathrm{SO}_{2}\right)=2.9 \times 10^{14} \mathrm{~cm}^{-2}$. No systematic differences in excitation temperature or column densities are found if the JCMT and CSO data are fitted separately, indicating that the absolute calibrations are consistent. Searches for ${ }^{34} \mathrm{SO}_{2}$ were negative to limiting values of $T_{\mathrm{MB}} \leq 0.075$ $\mathrm{mK}$ and yield $\tau\left({ }^{32} \mathrm{SO}_{2}\right) \leq 1.5$. There is a weak but noticeable trend in the rotation diagram data which yields higher excitation temperatures for the highest lying transitions detected.

\subsubsection{Unidentified Lines}

Several features at the $3 \sigma$ level or higher were observed in the surveys which could not be assigned to known molecular transitions. In several cases the line was detected with two or more local oscillator settings, and a definite rest frequency was obtained. For other features, no sideband assignment is

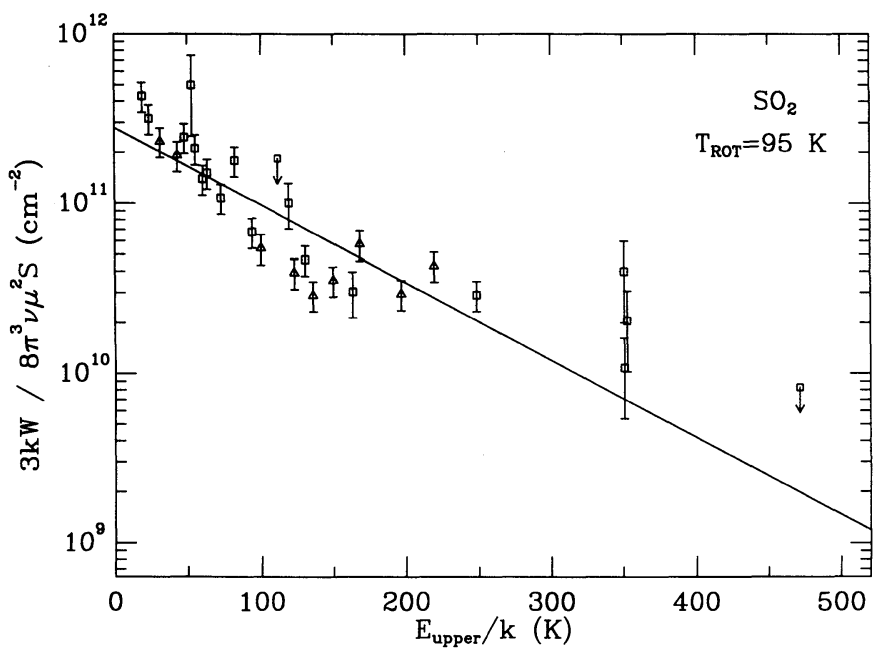

FIG. 7.-A rotation diagram plot for $\mathrm{SO}_{2}$. The data symbols are as in Fig. 6 
TABLE 6

UNIDENTIFIED LINES

\begin{tabular}{|c|c|c|c|c|c|}
\hline Species & $\begin{array}{c}\text { Frequency } \\
(\mathrm{MHz})\end{array}$ & $\underset{\left(\mathrm{K} \mathrm{km} \mathrm{s}^{-1}\right)}{T_{\mathrm{MB}} d V}$ & $\begin{array}{l}T_{\mathrm{MB}} \\
(\mathrm{K})\end{array}$ & $\begin{array}{c}\Delta V \\
\left(\mathrm{~km} \mathrm{~s}^{-1}\right)\end{array}$ & Telescope \\
\hline U.. & $213582 / 216839$ & 0.29 & 0.12 & 2.32 & JCMT \\
\hline U.. & $213838 / 216583$ & 0.55 & 0.093 & 5.57 & JCMT \\
\hline & $218508 / 221192$ & 0.47 & 0.27 & 1.62 & JCMT \\
\hline U... & 240086 & 0.50 & 0.09 & 5.18 & JCMT \\
\hline U........ & $240963 / 244036$ & 0.75 & 0.17 & 4.04 & JCMT \\
\hline $\mathrm{U} \ldots \ldots \ldots$ & $241531 / 244470$ & 0.40 & 0.10 & 3.77 & JCMT \\
\hline $\mathrm{U} \ldots \ldots \ldots$ & 243412 & 0.53 & 0.09 & 5.8: & JCMT \\
\hline U.. & 244589 & 0.84 & 0.10 & 7.55 & JCMT \\
\hline $\mathrm{U} \ldots \ldots \ldots$ & $245532 / 248468$ & 0.58 & 0.27 & 2.02 & JCMT \\
\hline U.......... & $261899 / 259101$ & 0.75 & 0.14 & 5.13 & JCMT \\
\hline $\mathrm{U} \ldots \ldots \ldots$ & $261965 / 259035$ & 1.35 & 0.26 & 4.80 & JCMT \\
\hline $\mathrm{U} \ldots \ldots \ldots$ & $313921 / 317319$ & 1.35 & 0.27 & 4.73 & JCMT \\
\hline U......... & $335062 / 337978$ & 0.44 & 0.21 & 1.97 & JCMT \\
\hline $\mathrm{U} \ldots \ldots \ldots$ & $335069 / 337972$ & 1.06 & 0.21 & 4.77 & JCMT \\
\hline U......... & $335250 / 337789$ & 0.85 & 0.19 & 4.18 & JCMT \\
\hline U........ & $335259 / 337781$ & 0.58 & 0.25 & 2.12 & JCMT \\
\hline $\mathrm{U} \ldots \ldots \ldots$ & 340054 & 1.82 & 0.38 & 4.37 & $\mathrm{CSO}$ \\
\hline U......... & 343087 & 1.32 & 0.18 & 6.89 & $\mathrm{CSO}$ \\
\hline $\mathrm{U} \ldots \ldots \ldots$ & 342522 & 1.52 & 0.37 & 3.88 & $\mathrm{CSO}$ \\
\hline $\mathrm{U} \ldots \ldots \ldots$ & 342731 & 2.08 & 0.33 & 5.93 & $\mathrm{CSO}$ \\
\hline U......... & 343003 & 0.78 & 0.17 & 4.38 & $\mathrm{CSO}$ \\
\hline U......... & 343058 & 1.32 & 0.20 & 5.98 & CSO \\
\hline U.......... & 356755 & 2.15 & 0.42 & 4.82 & CSO \\
\hline U........ & 398989 & 1.07 & 0.15 & 6.8: & $\mathrm{CSO}$ \\
\hline
\end{tabular}

possible and we have listed both frequencies compatible with the observed emission in Table 6.

\subsection{Excitation Calculations}

Although the rotation diagram method is useful for roughly constraining the excitation temperatures, it provides little information on the actual physical conditions, such as densities and kinetic temperatures. Moreover, for diatomic and linear polyatomic species for which only two or three lines are observed, the rotation diagram method gives very uncertain column densities. We have therefore performed full statistical equilibrium/radiative transfer calculations for a number of species, $\mathrm{CS}, \mathrm{HCS}^{+}, \mathrm{SiO}, \mathrm{H}_{2} \mathrm{CS}$, and OCS. The escape probability method adopted has been discussed in detail by Jansen, van Dishoeck, \& Black (1994) and van Dishoeck et al. (1993). Collisional and radiative excitation due to the $2.7 \mathrm{~K}$ cosmic background radiation field were taken into account. Although the far-infrared radiation by the $40 \mathrm{~K}$ ambient dust is considerable in IRAS 16293, in test calculations on several species it was found not to affect significantly the excitation of the rotational levels within the lowest vibrational state and was therefore neglected.

An essential ingredient in the calculations are the collisional rate coefficients. For $\mathrm{CS}$ and $\mathrm{SiO}$, we used the rate coefficients provided by Green (1991; as cited in Turner et al. 1992) interpolated to the appropriate temperature, while for OCS the rates of Broquier et al. (1986) were employed. In the case of $\mathrm{HCS}^{+}$we adopted the rates of Monteiro (1984) scaled for the difference in mass between $\mathrm{He}$ and $\mathrm{H}_{2}$. Finally, for $\mathrm{H}_{2} \mathrm{CS}$, we used the same rates as computed by Green (1991) for $\mathrm{H}_{2} \mathrm{CO}$ up to high temperatures, scaled to the $\mathrm{H}_{2} \mathrm{CS}-\mathrm{H}_{2}$ mass. Even though the levels within one $K_{p}$ ladder lie closer in energy in $\mathrm{H}_{2} \mathrm{CS}$, this molecule also has a lower dipole moment than $\mathrm{H}_{2} \mathrm{CO}$, which may tend to cancel any enhancements in the rates due to the former effect.

The resulting line ratios are presented in Figures 8 and 9 as functions of kinetic temperature and $\mathrm{H}_{2}$ volume density. In Figure 8, the ratios of a number of lines of linear rotors are shown, which are mostly sensitive to the density in the source. The observed values in IRAS 16293 for the optically thin isotopic species are indicated with the dashed lines and are seen to be remarkably consistent with a density of $(0.5-1) \times 10^{7} \mathrm{~cm}^{-3}$. Figure 9 contains several line ratios of $\mathrm{H}_{2} \mathrm{CS}$. Just like $\mathrm{H}_{2} \mathrm{CO}$, this molecule is a particularly useful diagnostic of both temperature and density (Jansen et al. 1993; van Dishoeck et al. 1993; Mangum \& Wootten 1993). Because it is a near-prolate rotor, radiative transitions within each $K_{p}$ ladder are rapid, but transitions between the various $K_{p}$ ladders are forbidden. Thus, the relative populations of the different $K_{p}$ ladders are controlled by collisions and are sensitive to temperature, whereas the relative populations within each ladder are mostly determined by the density. These trends are clearly seen in the ratios plotted in Figure 9 for both ortho- and para- $\mathrm{H}_{2} \mathrm{CS}$. The kinetic temperature is found to be $75 \pm 15 \mathrm{~K}$, and the density is about $10^{7} \mathrm{~cm}^{-3}$, consistent with the above estimates. The best fitting molecular column densities for $T=80 \mathrm{~K}$ and $n\left(\mathrm{H}_{2}\right)=8 \times 10^{6}$ $\mathrm{cm}^{-3}$ are included in Table 7 .

\section{DISCUSSION}

From the results of extensive interferometric and single-dish mapping at centimeter, millimeter, and far-infrared wavelengths, Mundy et al. (1990) have constructed a detailed model of the physical structure of IRAS 16293-2422. They delineate regions which they term the static core, outer disk, disk, and outflow. The static core is part of the larger scale $\rho$ Oph molecular cloud and is characterized by kinetic temperatures of $15-20 \mathrm{~K}$ and densities near $(1-2) \times 10^{4} \mathrm{~cm}^{-3}$. In the analysis of Mundy et al., the velocity patterns of the (colder) outer disk and (warmer) disk were interpreted as rotation about a central, accreting YSO. Physical conditions in the outer disk and disk were estimated to be $T_{\mathrm{KIN}}=15-20 \mathrm{~K}, n_{\mathrm{H}_{2}}=2 \times 10^{5} \mathrm{~cm}^{-3}$ and $40 \mathrm{~K}, \geq 5 \times 10^{7} \mathrm{~cm}^{-3}$ over scales of 10,000 and $1000 \mathrm{AU}$, respectively. The outflow patterns from IRAS $16293-2422$ are remarkably complex, possessing at least four distinguishable lobes up to $10^{\prime}$ in size with varying degrees of collimation (Walker et al. 1988; Mizuno et al. 1990). The outflow(s) has been probed at the 10-50 AU size scale by VLA observations of $1.3 \mathrm{~cm}$ water masers, which are found only near the southeastern continuum source (Wilking \& Claussen 1987; Wootten 1989; Terebey, Vogel, \& Myers 1992).

Subsequent high-resolution millimeter interferometry by Mundy et al. (1992) and Walker, Carlstrom, \& Bieging (1993) has resolved the disk into the binary components first detected by Wootten (1989) at centimeter wavelengths. In the latest 1.4 $\mathrm{mm}$ interferometric dust continuum images (Mundy et al. 1994) the truly circumstellar disks are at most 1 ".5 (250 AU) in diameter, while larger scale single-dish mapping of the dust continuum has detected a cold, massive core some $30^{\prime \prime}-40^{\prime \prime}$ in size (Mezger et al. 1992). The density in the immediate circumstellar region is at least $10^{9} \mathrm{~cm}^{-3}$. Accretion of this mass is inevitable, and accretion rates of $\dot{M} \sim 10^{-5} M_{\odot} \mathrm{yr}^{-1}$ are able to account entirely for the source luminosity. We therefore prefer to partition IRAS $16293-2422$ into ambient molecular cloud, circumbinary envelope, circumstellar disk(s), and outflow components. These boundaries are, of course, artificial in the sense that the true density and kinematic profile of the source is continuous, but they provide a useful starting point.

The varying physical properties of these regions lead to a range of molecular emission features, and each is visible in our 

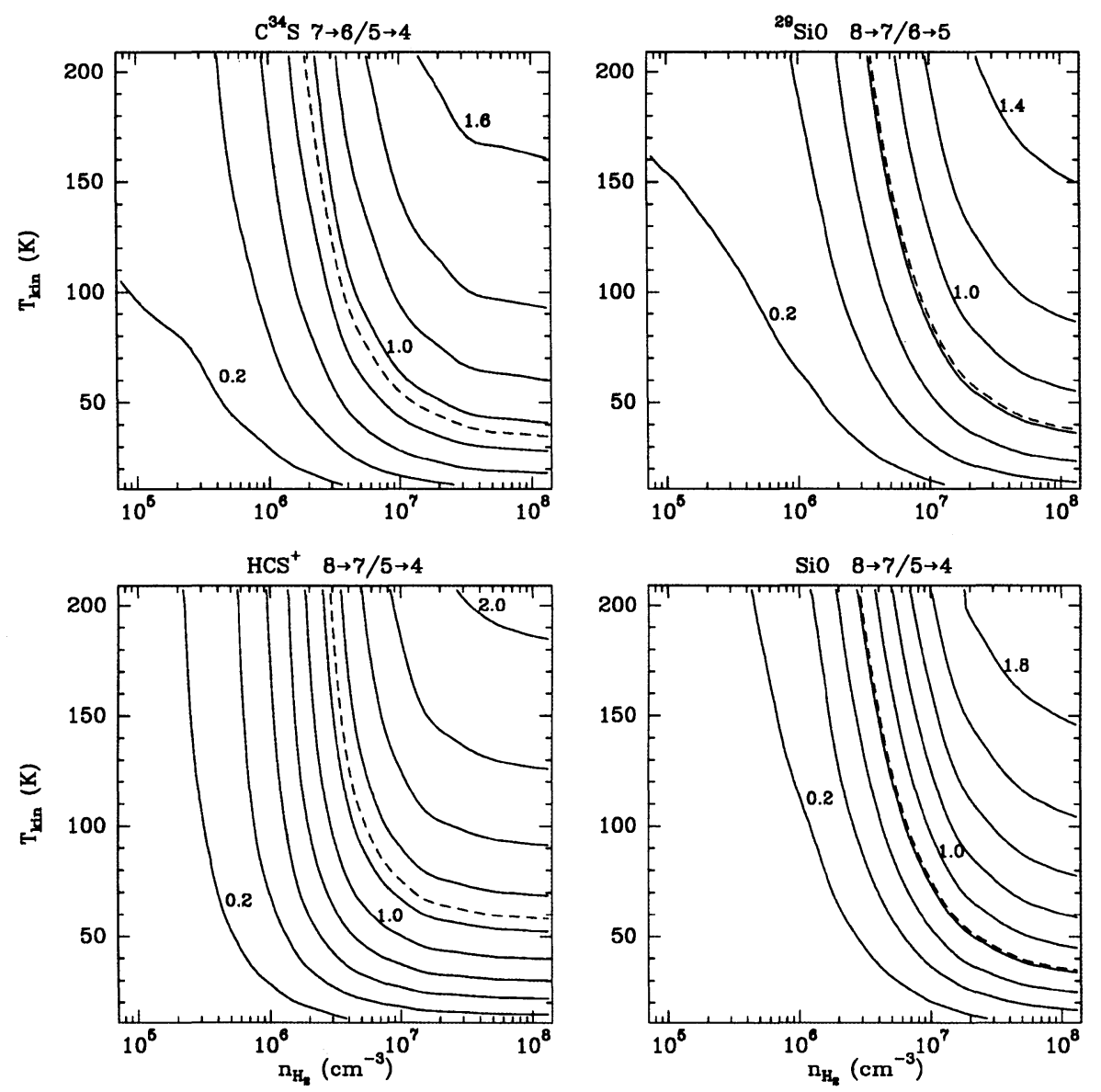

Fig. 8.-Pictorial outlines of the results of full statistical equilibrium/radiative transfer calculations for the $\mathrm{CS}$, $\mathrm{SiO}$, and $\mathrm{HCS}^{+}$molecules. In each segment, the ratios of selected molecular transitions are presented as contour diagrams with kinetic temperature and $\mathrm{H}_{2}$ volume density as variables. The observed ratios for the isotopically substituted species are presented as dashed lines. The lower $\mathrm{SiO}$ plot contains only ${ }^{28} \mathrm{SiO}$ data. The plots are presented for $\mathrm{CS}, \mathrm{SiO}$, and $\mathrm{HCS}{ }^{+}$column densities of $1 \times 10^{14}, 5 \times 10^{12}$, and $4 \times 10^{12} \mathrm{~cm}^{-2}$ and line widths of 4,5 , and $4 \mathrm{~km} \mathrm{~s}^{-1}$.

TABLE 7

Molecular Column Densities and Abundances

\begin{tabular}{|c|c|c|c|c|c|}
\hline \multirow[b]{2}{*}{ SPECIES } & \multicolumn{2}{|c|}{$N\left(\mathrm{~cm}^{-2}\right)$} & \multicolumn{3}{|c|}{$X=N / N\left(\mathbf{H}_{2}\right)$} \\
\hline & Rotation Diagram & Excitation Calculation & IRAS $16293^{a}$ & $\mathrm{~L} 134 \mathrm{~N}^{\mathrm{b}}$ & Orion Plateau ${ }^{b}$ \\
\hline $\mathrm{C}^{18} \mathrm{O}$ & & $1.8(16)$ & $1.0(-7)$ & $2(-7)$ & $2(-7)$ \\
\hline $\mathrm{C}^{17} \mathrm{O}$ & $(4.8 \pm 2.0)(15)$ & $7.5(15)$ & $3.8(-8)$ & $4(-8)$ & $4(-8)$ \\
\hline CS ... & $(8.5 \pm 4.0)(13)$ & & $1.1(-9)^{\mathrm{c}}$ & $7(-10)$ & $2(-8)$ \\
\hline ......... & $(1.5 \pm 0.6)(13)$ & $1.0(13)$ & & & \\
\hline SO ... & $(3.1 \pm 1.0)(14)^{\mathrm{d}}$ & $\ldots$ & $3.9(-9)^{\mathrm{c}}$ & $2(-8)$ & $5(-7)$ \\
\hline${ }^{34} \mathrm{SO} .$. & $(3.5 \pm 1.0)(13)$ & $\ldots$ & & & \\
\hline $\mathrm{SiO} \ldots \ldots \ldots \ldots \ldots$ & $(6.2 \pm 2.0)(12)^{d}$ & $\ldots$ & $1.0(-10)^{\mathrm{c}}$ & $<4(-12)$ & $(0.3-2)(-7)$ \\
\hline${ }^{29} \mathrm{SiO}$. & $(2.0 \pm 1.0)(12)$ & $1.0(12)$ & & & \\
\hline SiS . & $\leq 8(12)^{\mathrm{e}}$ & & $<4(-11)$ & $<6(-11)^{f}$ & $2(-8)^{\mathrm{g}}$ \\
\hline $\mathrm{HCS}^{+}$ & $(5.0 \pm 2.5)(12)$ & $4.0(12)$ & $2.0(-11)$ & $1(-10)$ & \\
\hline OCS .. & $(1.9 \pm 1.0)(14)^{d}$ & & $7.1(-9)^{\mathrm{c}}$ & $7(-9)$ & $6(-8)$ \\
\hline $\mathrm{OC}^{34} \mathrm{~S} \ldots \ldots$ & $(6.3 \pm 3.0)(13)$ & $6.3(13)$ & $\ldots$ & $\ldots$ & $\ldots$ \\
\hline $\mathrm{O}^{13} \mathrm{CS} \ldots \ldots \ldots \ldots$ & $(8.8 \pm 4.0)(13)$ & $5.6(13)$ & $\ldots$ & $\ldots$ & $\ldots$ \\
\hline${ }^{18}$ OCS $\ldots \ldots \ldots \ldots$ & & $<3.0(13)$ & $\ldots$ & & \\
\hline $\mathrm{H}_{2} \mathrm{~S} \ldots \ldots \ldots \ldots$ & $(3.0 \pm 0.5)(14)^{\mathrm{e}}$ & & $1.5(-9)$ & $8(-10)$ & $(0.1-4)(-6)$ \\
\hline $\mathrm{H}_{2} \mathrm{CS} \ldots \ldots \ldots$ & $(3.7 \pm 1.3)(13)$ & $3.4(13)$ & $1.7(-10)$ & & $\ldots$ \\
\hline $\mathrm{SO}_{2} \ldots \ldots \ldots \ldots \ldots$ & $(2.9 \pm 0.3)(14)$ & $\ldots$ & $1.5(-9)$ & $2(-9)$ & $5(-7)$ \\
\hline
\end{tabular}

a Using $N\left(\mathrm{H}_{2}\right)=2 \times 10^{23} \mathrm{~cm}^{-2}$ in a $20^{\prime \prime}$ beam, derived assuming $\mathrm{C}^{17} \mathrm{O}=3.8(-8)$.

${ }^{b}$ See van Dishoeck et al. (1992b) for references to observations.

c Derived from isotopic column densities assuming normal isotope ratios.

d Optically thick; rotation diagram results suspicious.

e Assuming $T_{\text {ex }}=30-100 \mathrm{~K}$.

f From Ziurys 1991 for TMC-1.

8 From Ziurys 1991, assuming $N\left(\mathrm{H}_{2}\right) \approx 5 \times 10^{22} \mathrm{~cm}^{-2}$ for the Orion Plateau. 

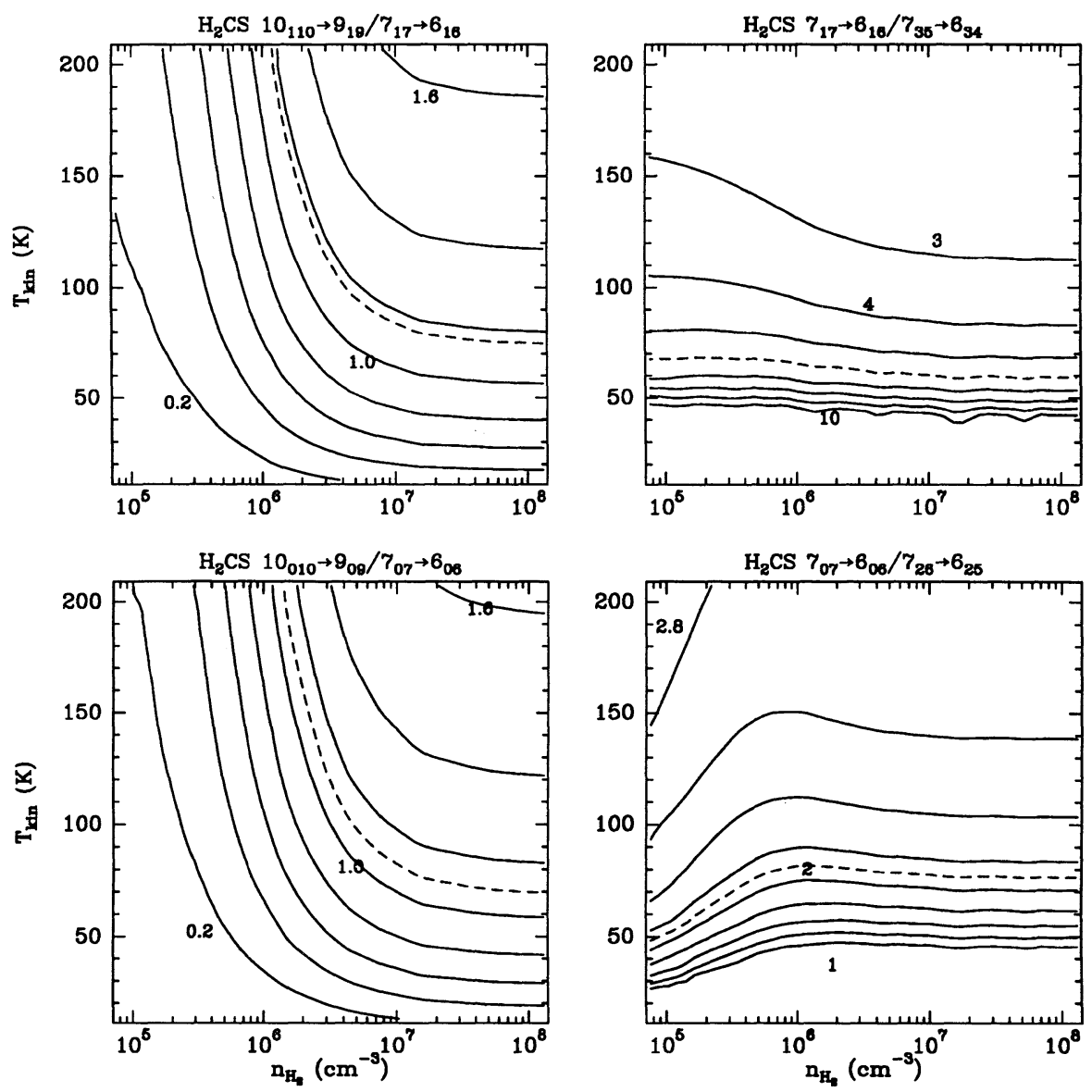

FiG. 9.-Radiative transfer plots as in Fig. 8, but for selected transition ratios in the ortho- and para- forms of $\mathrm{H}_{2} \mathrm{CS}$. Note the relative insensitivity to density of the ratios of different $K$ values in the same $J$ states (right), and the highly density sensitive nature of the cross- $J$ ratios in different $J$ states (left). Column densities of 1 and $3 \times 10^{14} \mathrm{~cm}^{-2}$ were used for ortho- and para- $\mathrm{H}_{2} \mathrm{CS}$, the line width was $\Delta V=4 \mathrm{~km} \mathrm{~s}^{-1}$.

spectral line surveys. Molecules are excellent physical probes of their local surroundings, but, as noted above, the rotation diagram approach provides very little in the way of physical diagnostics. We therefore use only Figures 8 and 9 to constrain the physical conditions in the emitting regions. Remarkably, each of the $\mathrm{Si}$ - and S-bearing molecules for which we have sufficient data to construct full statistical equilibrium calculations seems to arise from a region whose kinetic temperature is approximately $70-80 \mathrm{~K}$, with a density of some $(0.5-1.0)$ $\times 10^{7} \mathrm{~cm}^{-3}$. The densities are similar to, but the kinetic temperatures are much in excess of, those obtained for the central regions of IRAS 16293 in previously constructed models of this source. The only evidence for $\mathrm{Si}$ - and S-bearing species in the cold, moderate density ambient molecular cloud is the selfabsorption present in the CS $5 \rightarrow 4$ spectrum, but this region is quite evident in the more volatile species examined by van Dishoeck et al. (1994). There is a weak trend in the data for those molecules with the most extended emission (e.g., CS) to have lower excitation temperatures and densities than those with the most compact emission (e.g., SiO), but, again, all of the inferred kinetic temperatures are considerably hotter than any dust component of the spectral energy distribution.

In Table 7 we summarize the beam-averaged column densities and abundances obtained by the LTE or full statistical equilibrium calculations. As noted above, the latter calcu- lations produce somewhat lower column densities than the rotation diagram approach, since the populations of higher levels are calculated self-consistently. The absolute abundances are obtained by scaling with respect to an assumed $\mathrm{C}^{17} \mathrm{O}$ fraction of $3.8 \times 10^{-8}$, that is, we use optically thin $\mathrm{CO}$ emission to trace the bulk of the mass distribution. Clearly, this is an overly simplistic interpretation, and a more detailed analysis of the chemical and physical structure of this source will be reported elsewhere. Nevertheless, a number of general features are immediately apparent.

Namely, the sulfur chemistry toward IRAS 16293 appears to be intermediate between the composition found toward lowmass star-forming regions such as TMC-1 and L134N on the one hand, and high-mass star-formation regions such as the plateau and hot core sources of Orion. For example, the CS and SO abundances are similar in the three low-luminosity sources, but the $\mathrm{H}_{2} \mathrm{~S}, \mathrm{SO}_{2}$, and $\mathrm{SiO}$ abundances toward IRAS 16293 are at least an order of magnitude larger than found in Taurus cores, including YSOs such as L1551. Further, the similar $\mathrm{H}_{2} \mathrm{~S}$ and $\mathrm{SO}_{2}$ abundances in L134N are found only at the "sulfur/oxygen" peak in this source (Swade 1989). The absolute enhancements in the plateau source of Orion are much larger than those observed here, but the relative abundances of $\mathrm{SO}, \mathrm{SiO}, \mathrm{SO}_{2}$, and $\mathrm{H}_{2} \mathrm{~S}$ show interesting parallels. Indeed, if the source sizes of $\mathrm{SiO}, \mathrm{SO}$, and OCS are signifi- 
cantly smaller than that of $\mathrm{CO}$, the absolute abundances in IRAS 16293 would be correspondingly higher and approach those seen in Orion KL.

The CO and CS characteristics are similar to those found in previous studies of IRAS 16293, and arise from a source some $10^{\prime \prime}-15^{\prime \prime}$ in size at roughly $40 \mathrm{~K}$ - the disk source of Mundy et al. (1990). Does the considerably smaller source size derived for the Si- and S-species, which in some sense trace higher temperature or more energetic regions of the cloud, indicate high temperature processes in circumstellar gas close to the young stars or outflowing gas as is traced by the water masers? Temperatures near $100 \mathrm{~K}$ are reached only in the inner few AU of most disk models with accretion rates near $10^{-5} M_{\odot} \mathrm{yr}^{-1}$, and such a small source would not be expected to emit lines of several K, as is associated with SO for example. Further, it has been argued that the substantial optical depth of the source at $30 \mu \mathrm{m}$ is provided by a nearly edge-on geometry in which the colder, outer regions of the flaring circumstellar disk block out light from the warmer, inner regions of the disk which have substantial optical depths in the dust at submillimeter wavelengths (Mundy et al. 1992; Beckwith et al. 1990).

These features coupled with the supersonic line wings, high kinetic temperatures, and critical densities for the gas with little evidence of dust emission at the same temperature are consistent with a shock origin for much of the sulfur/silicon chemistry observed in this source. For example, an $80 \mathrm{~km} \mathrm{~s}^{-1}$ shock propagating into a medium with $n=10^{5} \mathrm{~cm}^{-3}$ is capable of generating column densities near $10^{22} \mathrm{~cm}^{-2}$ of post-shocked gas with kinetic temperatures of $\geq 100 \mathrm{~K}$ (Hollenbach, Chernoff, \& McKee 1989) and compression factors of $\sim 100$. The shock-heated dust grains are only weakly coupled to the gas and therefore have much lower temperatures, thereby contributing only weakly to the observed emission from dust. Speeds near $80 \mathrm{~km} \mathrm{~s}^{-1}$ are chosen merely to indicate the plausibility of shock-generated warm gas in this source. High-velocity bipolar winds from YSOs typically have velocities well in excess of this value, and the VLA water maser observations of Wootten (1989) and Terebey et al. (1992) reveal a morphology reminiscent of the optical jets seen around $T$ Tauri stars. The observed $\mathrm{CO} 2 \rightarrow 1$ and $3 \rightarrow 2$ supersonic lines wings yield a column density of roughly $3 \times 10^{21} \mathrm{~cm}^{-2}$ for the outflowing gas.

Of the many species listed in Table 7, only $\mathrm{H}_{2} \mathrm{~S}$ and perhaps $\mathrm{SO} / \mathrm{SO}_{2}$ show the tremendous variability observed for $\mathrm{SiO}$. Recent shock models favor a J-type origin for $\mathrm{SiO}$ due to the increased sputtering and grain destruction rates in such shocks, while sulfur-bearing species, especially $\mathrm{H}_{2} \mathrm{~S}$, are formed more abundantly in C-type shocks (Neufeld \& Dalgarno 1989). Detailed models of the physical structure of outflows surrounding YSOs are only now becoming available, and it is not well established whether environments ranging from C-type through J-type shocks can be generated for low-mass objects such as IRAS 16293. Details such as the magnetic field and density structure in collapsing molecular cloud cores can profoundly influence shock physics and chemistry both temporally and spatially but remain poorly characterized.

The contribution of grain mantle chemistry to the abundances of species such as $\mathrm{H}_{2} \mathrm{~S}$ also awaits further investigation. Interestingly, little evidence for substantial grain mantle depletion is found in our single dish surveys despite the very high densities and cool dust temperatures derived for this source. Toward IRAS 16293-2422 itself, the total mass inferred from the " canonical" $\mathrm{CO}$ abundance of $10^{-4}$ is in good accord with that determined from dust continuum observations if the interstellar opacity of Hildebrand (1983) is combined with a $\lambda^{-1.5}$ emissivity law (Mundy et al. 1994). However, interferometric results indicate considerable complexity on smaller size scales. Both $\mathrm{NH}_{3}$ and $\mathrm{SO}$, for example, show abundance variations of at least a factor of 10 over the high column density region outlined by the millimeter dust continuum, with the northeastern source being particularly weak in SO and $\mathrm{C}^{\mathbf{1 8}} \mathrm{O}$. Further observations at very high spatial and spectral resolution will be required to address these important cosmochemical issues.

\section{SUMMARY}

Emission from a variety of $\mathrm{Si}$ - and $\mathrm{S}$-bearing molecules has been detected in IRAS $16293-2422$. While some of these molecules have abundances similar to the surrounding molecular cloud which gave rise to the new stars, other species have clearly been affected by the star-formation process. In particular, the refractory $\mathrm{SiO}$ species appears to be significantly enhanced, possibly as the result of interaction between the bipolar outflow(s) from the protobinary and the surrounding circumbinary envelope. No compelling evidence of widespread depletion of molecules onto grain mantles in this high-density molecular cloud core has been found, but interferometric images demonstrate that such processes do occur in the immediate circumstellar environment. The longer evolutionary timescales of low-mass star formation may therefore enable both outflow and accretionary processes to modify the chemistry in modest luminosity YSOs simultaneously.

The authors are grateful to John Black for discussions on the excitation of various molecules in this source. G. A. B. would like to acknowledge support from NASA (NAGW-2297), as well as the David and Lucille Packard and Alfred P. Sloan Foundations, while work on astrochemistry in Leiden is supported by a PIONIER grant from the Netherlands Organization for Scientific Research (NWO). L. G. M. would like to acknowledge support from NASA (NAGW-3066).

\section{REFERENCES}

Beckwith, S., Sargent, A. I., Chini, R., \& Güsten, R. 1990, AJ, 99, 924

Black, J. H. 1993, private communication

Black, J. H., \& Willner, S. P. 1984, ApJ, 279, 673

Blake, G. A., Sutton, E. C., Masson, C. R., \& Phillips, T. G. 1987, ApJ, 315, 621

Broquier, M., Picardbersellini, A., Whitaker, B. J., \& Green, S. 1986, J. Chem. Phys., 84, 2104

Brown, P. D., \& Charnley, S. B. 1990, MNRAS, 244, 432

Caselli, P., Hasegawa, T. I., \& Herbst, E. 1993, ApJ, 408, 548

d'Hendecourt, L. B., Allamandola, L. J., \& Greenberg, J. M. 1985, A\&A, 152, 130

Green, S. 1991, ApJS, 76, 879

Groesbeck, T. D. 1994, Ph.D. thesis, California Inst. of Technology
Hartquist, T. W., Rawlings, J. M. C., Williams, D. A., \& Dalgarno, A. 1993, QJRAS, 34, 213

Helmich, F. P., Jansen, D. J., de Graauw, T., Groesbeck, T. D., \& van Dishoeck, E. F. 1994, A\&A, in press

Hildebrand, R. H. 1983, QJRAS, 24, 267

Hollenbach, D. J., Chernoff, D. F., \& McKee, C. F. 1989, in Infrared Spectroscopy in Astronomy, 22d Eslab Symposium, ed. B. H. Kaldeich (ESA SP-290), (Noorwijk: ESTEC), 245

Jansen, D. J., van Dishoeck, E. F., \& Black, J. H. 1994, A\&A, 282, 605

Jenkins, E. B. 1987, in Interstellar Processes, ed. D. A. Hollenbach \& H. A. Thronson (Dordrecht: Reidel), 533

Kutner, M. L., \& Ulich, B. L. 1981, ApJ, 250, 341 
Lada, C. J. 1991, in The Physics of Star Formation and Early Stellar Evolution, ed. C. J. Lada \& N. D. Kylafis (Dordrecht: Kluwer), 329

Langer, W. H., \& Penzias, A. A. 1990, ApJ, 357, 477

Mangum, J., \& Wootten, A. 1993, ApJS, 89, 123

Mezger, P. G., Sievers, A., Zylka, R., Haslam, C. G. T., Kreysa, E., \& Lemke, R. 1992, A\&A, 265, 743

' Mizuno, A., Fukui, Y., Nozawa, S., \& Takano, T. 1990, ApJ, 356, 184

Monteiro, T. 1984, MNRAS, 210, 1

- Menten, K. M., Serabyn, E., Güsten, R., \& Wilson, T. L. 1987, A\&A, 177, L57

Mundy, L. G., Wilking, B. A., \& Myers, S. T. 1986, ApJ, 311, 275

Mundy, L. G., Wootten, H. A., \& Wilking, B. A. 1990, ApJ, 352, 159

Mundy, L. G., Wootten, H. A., Wilking, B. A., Blake, G. A., \& Sargent, A. I. 1992, ApJ, 385, 306

Mundy, L. G., et al. 1994, in preparation

Neufeld, D. A., \& Dalgarno, A. 1989, ApJ, 340, 869

Poynter, R. L., \& Pickett, H. M. 1992, NASA JPL Publ. 80-23, revision 3

Rawlings, J. M. C., Hartquist, T. W., Menten, K. M., \& Williams, D. W. 1992, MNRAS, 255, 471

Shu, F. H. 1992, in Protostars \& Planets III, ed. E. H. Levy \& J. Lunine (Tucson: Univ. Arizona Press), 3

Sutton, E. C., Jaminet, P. A., Danchi, W. C., \& Blake, G. A. 1991, ApJS, 77, 255
Swade, D. A. 1989, ApJ, 345, 828

Terebey, S., Vogel, S. N., \& Myers, P. C. 1992, ApJ, 390, 181

Turner, B. E., Chen, K-W., Green, S., \& Lubowich, D. A. 1992, ApJ, 399, 114

van Dishoeck, E. F., Blake, G. A., Draine, B. T., \& Lunine, J. I. 1992b, in Protostars \& Planets III, ed. E. H. Levy \& J. Lunine (Tucson: Univ. Arizona Press), 163

van Dishoeck, E. F., Blake, G. A., Jansen, D. J., \& Groesbeck, T. D. 1994, ApJ, in preparation

van Dishoeck, E. F., Glassgold, A. E., Guélin, M., Jaffe, D. T., Neufeld, D. A. Tielens, A. G. G. M., \& Walmsley, C. M. 1992a, in IAU Symp. 150, Astrochemistry of Cosmic Phenomena, ed. P. D. Singh (Dordrecht: Kluwer), 285 van Dishoeck, E. F., Jansen, D. J., \& Phillips, T. G. 1993, A\&A, 279, 541

Walker, C. K., Carlstrom, J. E., \& Bieging, J. H. 1993, ApJ, 402, 655

Walker, C. K., Carlstrom, J. E., Bieging, J. H., Lada, C. H., \& Young, E. T. 1990, ApJ, 364, 173

Walker, C. K., Lada, C. H., Young, E. T., Maloney, P. R., \& Wilking, B. A 1986, ApJ, 309, L47

Walker, C. K., Lada, C. H., Young, E. T., \& Margulis, M. 1988, ApJ, 332, 335

Wilking, B. A., \& Claussen, M. J. 1987, ApJ, 320, L133

Wootten, A. 1989, ApJ, 337, 858

Ziurys, L. M. 1991, ApJ, 379, 260 